\title{
A Novel Method for Fitting Unimodal Continuous Distributions on a Bounded Domain Utilizing Expert Judgment Estimates
}

\author{
Samuel Kotz ${ }^{1}$ and J. René van Dorp ${ }^{2}$ \\ The George Washington University, USA
}

\begin{abstract}
Recent advances in computation technology for simulation/uncertainty analyses have shed new light on the triangular distribution and its use to describe the uncertainty of bounded input phenomena. Herein, we develop a novel fitting procedure for a continuous unimodal (four-parameter) family of distributions on a bounded domain, utilizing three properly selected quantile estimates and an estimate of the most likely value. The family in question is the two-sided power (TSP) family of which the triangular distribution is a member. We analyze some of the procedure's fitting characteristics and use them to estimate the waiting time distribution in a stationary $\mathrm{M} / \mathrm{G} / 1$ queuing system and the completion time distribution of a small project network example taken from the shipbuilding domain.
\end{abstract}

\section{INTRODUCTION}

The triangular distribution is one of the first continuous distributions proposed back in 1755 by English mathematician Thomas Simpson. It received widespread attention only as late as the 1960's

\footnotetext{
1 Samuel Kotz, Department of Engineering Management and Systems Engineering, School of Engineering and Applied Science, The George Washington University, 1776 G Street, N.W. , Suite 110, Washington D.C. 20052. E-mail: kotz@gwu.edu.

2 J. René van Dorp, Corresponding Author. Same address. Phone: 202-994-6638, Fax: 202-994-0245, E-mail: dorpjr@gwu.edu.
} 
in the context of the Project Evaluation and Review Technique (PERT) as an alternative to the fourparameter beta distribution

$$
\begin{aligned}
& f_{T}(t \mid a, b ; \alpha, \beta)=\frac{\Gamma(\alpha+\beta)}{\Gamma(\alpha) \Gamma(\beta)} \frac{(t-a)^{\alpha-1}(b-t)^{\beta-1}}{(b-a)^{\alpha+\beta-1}} \\
& a \leq t \leq b, \alpha>0, \beta>0
\end{aligned}
$$

which has some difficulties regarding the interpretation of its parameters $\alpha$ and $\beta$ (see, e.g., Clark 1962, Grubbs 1962, Moder and Rogers 1968, Elmaghraby 1978, Keefer and Verdini 1993 , Kamburowski 1997, Johnson 1997, Lau et al. 1998, and Herrerías et al. 2003, among others). In this paper we investigate a recent generalization of the triangular distribution (see, e.g., van Dorp and Kotz 2002a) known as the two-sided power (TSP) distribution with the probability density function (pdf)

$$
f_{X}(x \mid a, m, b, n)= \begin{cases}\frac{n}{(b-a)}\left(\frac{x-a}{m-a}\right)^{n-1} & a<x \leq m \\ \frac{n}{(b-a)}\left(\frac{b-x}{b-m}\right)^{n-1} & m \leq x<b,\end{cases}
$$

where $n>0$ is a real constant, as an alternative to the beta distribution when distributional parameters of a random bounded phenomenon are to be assessed via the expert judgment. For $n>1(0<n<1)$ the $\operatorname{pdf}(2)$ is unimodal (U-shaped provided $a<m<b)$. The cumulative distribution function (cdf) follows from (2) using straightforward manipulations:

$$
\operatorname{Pr}(X \leq x) \equiv F_{X}(x \mid a, m, b, n)=\left\{\begin{array}{cl}
\frac{(m-a)}{(b-a)}\left(\frac{x-a}{m-a}\right)^{n} & a \leq x \leq m \\
1-\frac{(b-m)}{(b-a)}\left(\frac{b-x}{b-m}\right)^{n} & m \leq x \leq b .
\end{array}\right.
$$

For $n=2(n=1)$ the $\operatorname{pdf}(2)$ reduces to a triangular (uniform) pdf. An additional advantage of the TSP family of distributions over that of the beta family for Monte Carlo analysis is that TSP distributions have a greater moment ratio coverage $\left(\theta_{1}, \theta_{2}\right)$ - at least for unimodal distributions (see, e.g., van Dorp and Kotz 2002b). Here, we have the squared skewness $\theta_{1}=\mu_{3}^{2} / \mu_{2}^{3}$, the kurtosis $\theta_{2}=\mu_{4} / \mu_{2}^{2}, E[X]=\mu_{1}$ and $\mu_{k}=E\left(X-\mu_{1}\right)^{k}$ (for $\left.k>2\right)$. Lau et al. (1998), among others, note a restricted coverage for the beta family as far as kurtosis $\theta_{2}$ is concerned. 
The unprecedented advances in quantitative methodology and their penetration into applied sciences and engineering during the last several decades (recall the, by now standard, tools such as @RISK by the Palisade Corporation, Crystal Ball by Decision Engineering, and ARENA by Rockwell Software) have reinvigorated the use of distributions with bounded support and resulted in a re-assessment of the scope and nature of an expert's activities. Experts are, as a rule, classified into two, usually unrelated, groups: 1) substantive experts (also known as technical experts or domain experts) who are knowledgeable about the subject matter at hand and 2) normative experts possessing knowledge of the appropriate quantitative analysis techniques (see, e.g., Pulkkinen and Simola 2000 for details). In the absence of data and in the context of simulation and uncertainty analyses, substantive experts are used (often by necessity) to specify these bounded input distributions.

In the last decade, integration of graphically interactive and statistical procedures for bounded input distribution modeling has become a topic of intensive research (see, e.g., DeBrota et al. 1989, AbouRizk et al. 1992 and Wagner and Wilson 1995, 1996) to facilitate their elicitation by experts. AbouRizk et al. (1992) have developed software with a graphical user interface (GUI) to ease fitting of beta distributions using a variety of methods and DeBrota et al. (1989) have developed one for fitting bounded Johnson $\mathrm{S}_{B}$ distributions. (A Johnson $\mathrm{S}_{B}$ random variable $Y$ with scale parameter $\gamma \in \mathbb{R}$ and shape parameter $\delta>0$ is by definition a transformed standard normal random variable $Z$ by means of the transformation $\left.Y=\{1+\exp [-(Z-\gamma) / \delta]\}^{-1}\right)$. Both methods require a specification of the lower and upper bounds of the distribution's support. Wagner and Wilson (1995, 1996) introduced univariate Bézier distributions (or curves), which are a variant of spline functions, and the software tool PRIME with a GUI to specify them. Bézier curves utilize a number of control points as its parameters where each control point is defined by two coordinates. Two of these control points define its lower and upper bounds. The system of Bézier distributions allows for great flexibility in input distribution modeling for stochastic simulations.

However, as Wagner and Wilson (1996) indicate, random variate generation from a Bézier distribution is, at present, computationally inefficient since its inverse cdf is not available in a closed 
form as it is the case for the beta or Johnson $S_{B}$ distributions. On the other hand from the TSP cdf (3) we immediately obtain its inverse:

$$
\begin{aligned}
& F_{X}^{-1}(y \mid a, m, b, n)= \\
& \left\{\begin{array}{cl}
a+\left\{y(m-a)^{n-1}(b-a)\right\}^{1 / n}, & \text { for } 0 \leq y \leq q \\
b-\left\{(1-y)(b-m)^{n-1}(b-a)\right\}^{1 / n}, & \text { for } q \leq y \leq 1 .
\end{array}\right.
\end{aligned}
$$

where

$$
q \equiv \operatorname{Pr}(X \leq m)=\frac{m-a}{b-a}
$$

represents the mode probability. This allows for straightforward and efficient sampling using the inverse cdf technique and a pseudo- random number generator (see, e.g., Banks et al. 2005). Observe that the mode probability $q$ in $(5)$ does not depend on the parameter $n$.

The expressions for the mean and variance of a TSP variable can directly be obtained from (2):

$$
E[X]=\frac{a+(n-1) m+b}{n+1}, \operatorname{Var}[X]=(b-a)^{2} \cdot\left\{\frac{n-2(n-1) \frac{m-a}{b-a} \frac{b-m}{b-a}}{(n+2)(n+1)^{2}}\right\} .
$$

The denominator $n+1$ in the expression for the mean in (6) may be interpreted as a virtual sample size when $n$ is not an integer (and as a sample size otherwise) of a sample with $n-1$ virtual observations of value $m$ and two additional ones with values $a$ and $b$. The notion of a non-integer virtual sample size corresponds to the notion of a non-integer prior sample size introduced by Ferguson (1973). Hence, one may indirectly elicit the shape parameter $n$ by asking an expert for the relative importance of the elicited most likely value $\widehat{m}$ (for assessing the average) compared to elicited bounds $\widehat{a}$ or $\widehat{b}$. Note that a value of $n-1=1$ (corresponding to a triangular distribution) indicates the same importance while values greater (less) than 1 indicate a larger (lesser) importance. The above elicitation approach utilizes a comparison approach similar to the popular paired comparison elicitation techniques in psychological scaling models (see, e.g., Cooke 1991). However, this concept of relative importance to elicit the shape parameter $n$ could be a challenge for a substantive expert to assess it cognitively. 
In the remainder of this paper we shall develop an alternative fitting (or elicitation) procedure for the four parameters $a, m, b$ and $n$ of a TSP distribution in the absence of data. In Section 2, we shall motivate the elicitation of a lower and upper quantiles $a_{p}$ and $b_{r}$ respectively, the most likely value $m$ and an additional quantile $x_{s}$ as the inputs for this procedure. In Section 3, we develop a numerical algorithm that solves for the TSP parameters $(a, m, b$ and $n)$ from $a_{p}, b_{r}, m$ and $x_{s}$ and derive boundary conditions for the additional quantile $x_{s}$ in terms of an asymmetric Laplace and uniform distributions which, if met, would guarantee feasibility. Two uncertainty analysis examples are presented in Section 4. The first one involves estimation of the marginal waiting time distribution in a stationary $M / G / 1$ queuing system and the second involves the estimation of the completion time distribution of a small project network. A concluding remark is provided in Section 5. The Appendix discusses some mathematical details regarding the algorithm designed in Section 3.

\section{MOTIVATION FOR THE ELICITATION OF THREE QUANTILES AND THE MODE} Johnson (1997) and Williams (1992) emphasize the intuitive appeal of the triangular distribution $(n=2$ in $(2)$ and $(3))$ amongst practitioners and engineers since its parameters $a, m$ and $b$ correspond to optimistic $(\widehat{a})$, most likely $(\widehat{m})$ and pessimistic $(\widehat{b})$ estimates, respectively, of an uncertain bounded phenomenon. A college text-book on discrete event simulation (Altiok and Melamed 2001) utilizing the popular simulation package ARENA specifically recommends the triangular distribution in the case when the underlying distribution is unknown, while a certain minimal $\widehat{a}$, some maximal $\widehat{b}$ and a most likely values $\widehat{m}$ are available. Other transparent properties of the triangular distribution are: (1) its parameters $a, m$ and $b$ are of the same dimension as the quantity of interest, and (2) the probability mass $q$ to the left of the most likely value $m$ equals the relative distance from the mode $m$ to the lower bound $a$ relative to the whole support $[a, b]$ (see, Eq. (5)). The properties above seem to allow for direct elicitation of triangular parameters and not to require visual interactive elicitation software similar to those developed for the beta, Johnson $S_{B}$ and Bézier distributions. 
From a statistical perspective, however, the elicitation of a minimal and maximal values $\widehat{a}$ and $\widehat{b}$ respectively from a substantive expert in modeling the uncertainty distribution of a bounded phenomenon has been of concern and objections to a number of analysts (see, e.g., Selvidge 1980, Davidson and Cooper 1980, Alpert and Raiffa 1982, Keefer and Verdini 1993), since these extreme values quite likely fall outside the realm of his/her experience in spite of his/her familiarity with the phenomenon. Consequently, early PERT practitioners have taken the liberty (and apparently with a good reason) to replace the lower bound estimate $\widehat{a}$ (upper bound estimate $\widehat{b})$ by the $p$-th $((1-p)$ th) percentile, setting $a_{p}=\widehat{a}\left(b_{1-p}=\widehat{b}\right)$. It has been verified during the last decades that assessment of percentiles in the vicinity of extreme values, such as the 0.01 and 0.99 percentiles (Alpert and Raiffa 1982) is also (similarly to the actual lower and upper bounds) quite often beyond our accumulated experience, since they too, as a rule, correspond to rather rare events (Keefer and Verdini 1993). The latter authors observe that the 0.10 and 0.90 quantiles have been found to be more reliable than the "extreme" 0.01 and 0.99 percentiles (Selvidge 1980) or even the "intermediate" 0.05 and 0.95 percentiles (Davidson and Cooper 1980).

It should however be noted that the specification of a lower quantile $a_{p}$, a most likely value $m$ and an upper quantile $b_{1-p}$ does not uniquely define a distribution for a bounded uncertain (random) input phenomenon in simulation/uncertainty analyses. Figure 1 depicts four different distributions that satisfy the constraints set by the lower percentile $a_{0.10}$ of 6.5 , the most likely value $m$ of 7 and the upper percentile $b_{0.90}$ of 10.5 . Note that these four distributions have substantial differences in their support. Table 1 provides the values for the mean, variance, skewness and kurtosis for these distributions. The largest value of the variance in Table 1 corresponds to the unimodal TSP distribution with parameter $n=7$, while the smallest one is obtained for the uniform distribution (which may be counterintuitive). On the other hand, the mean values in Table 1 behave in an opposite manner.

The differences amongst the distributions in Figure 1 and Table 1 are quite noticeable and would, no doubt, affect output results in various simulation/uncertainty analyses. This may present a dilemma to a normative expert regarding the type of distribution to be used. From this numerical 
example one concludes that eliciting a lower percentile $a_{p}$, a most likely estimate $m$ and an upper estimate $b_{1-p}$ is not sufficient to fully describe an input distribution. In the last 20 years a number of investigators arrived at a similar conclusion and suggested the elicitation of five (Alpert and Raiffa 1982), seven (Selvidge 1980) or even as many as nine quantiles (Lau et al. 1998) to achieve a proper assessment of solely the mean and the variance of the uncertain phenomenon rather than its complete distribution, which is required for simulation/uncertainty analyses. It would therefore seem reasonable to build on the intuitive appeal of the triangular distribution, when describing a distribution of a bounded input phenomenon, and specify what additional information needs to be provided by the substantive expert (in addition to the values of $a_{p}, m$ and $b_{1-p}$ ) to describe fully a $T S P(a, m, b, n)$ distribution $(2)$.
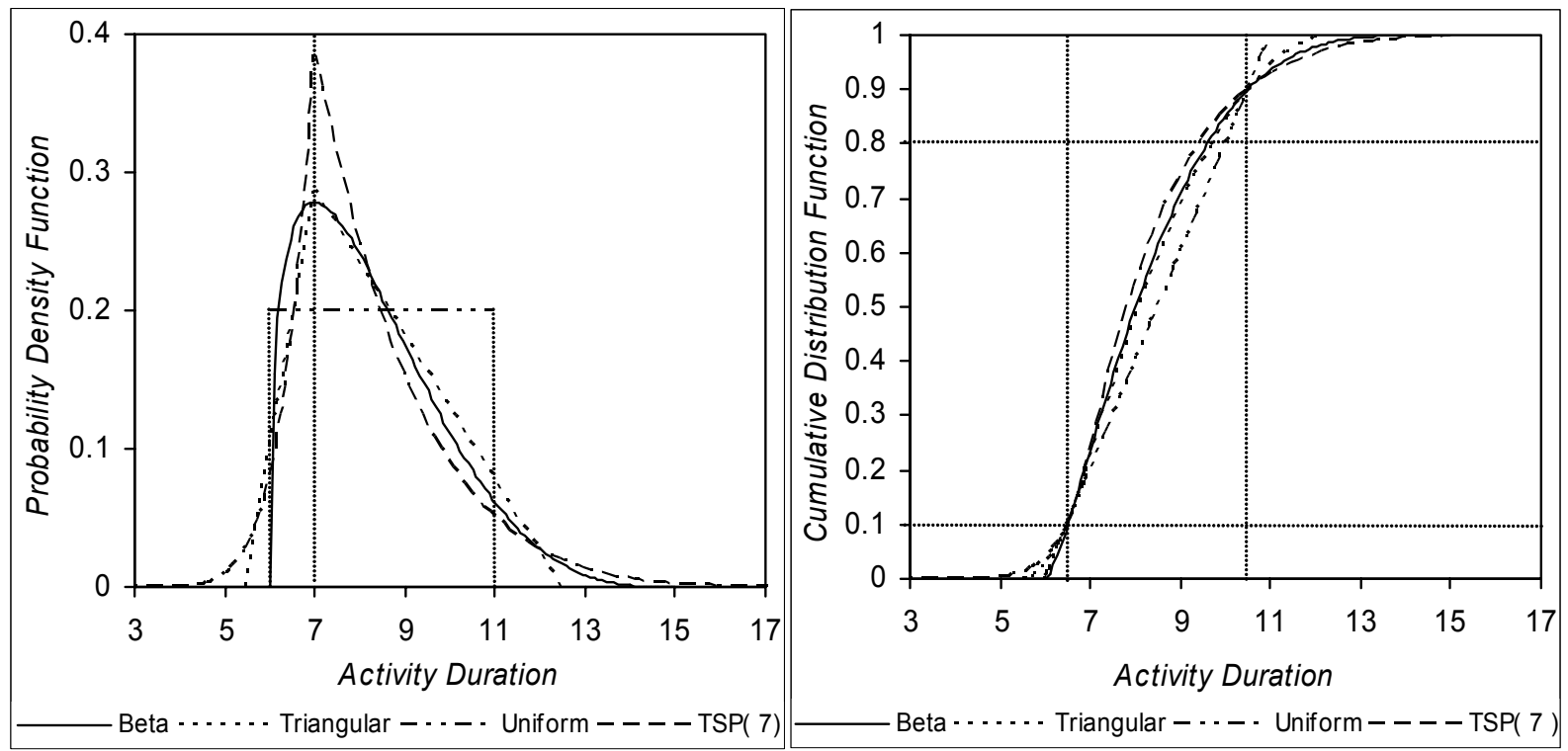

Figure 1: Beta, triangular, uniform and TSP distributions satisfying the constraints set by $a_{0.10}=6.5, m=7$, and $b_{0.90}=10.5$. Beta with parameters: $a=6, b=15$, $\alpha=1.384, \beta=4.071 ;$ Triangular: $a=5.464, m=7, b=12.452 ;$

Uniform: $a=6, b=11$; TSP: $a=2.687, m=7, b=20.891, n=7$. 
Table 1: The lower and upper bounds, mean, variance, skewness and kurtosis for the distributions in Figure 1.

\begin{tabular}{lcccc}
\hline & Beta & Uniform & Triangular & TSP $(7)$ \\
\hline Lower Bound & 6.00 & 6.00 & 5.46 & 2.69 \\
Upper Bound & 16.00 & 11.00 & 12.45 & 20.89 \\
Mean & 8.28 & 8.50 & 8.31 & 8.20 \\
Variance & 2.38 & 2.08 & 2.25 & 2.78 \\
Skewness & 0.60 & 0.00 & 0.21 & 1.25 \\
Kurtosis & 3.08 & 1.80 & 2.40 & 4.60 \\
\hline
\end{tabular}

In this paper, we propose to solve for the parameters $a, b, m$ and $n$ of a TSP distribution (2) from a lower quantile estimate $\widehat{a}_{p}$, most likely estimate $\widehat{m}$, an upper-quantile estimate $\widehat{b}_{1-p}$ and an additional quantile $\widehat{x}_{s}$ (to be determined also by a substantive expert) such that

$$
\widehat{a}_{p}<\widehat{x}_{s}<\widehat{b}_{1-p}
$$

Specification of an additional quantile $\widehat{x}_{s}$, satisfying $(7)$, allows for derivation of boundary

conditions utilizing asymmetric Laplace and uniform cdfs which, when satisfied, would guarantee the feasibility of a $\operatorname{TSP}(a, m, b, n)$ distribution. These boundary conditions may prove to be useful in an automated visually interactive elicitation procedure. In the next section, an algorithm is developed that solves for the TSP parameters $a, b, m$ and $n$ from the estimates $\widehat{a}_{p}, \widehat{m}, \widehat{b}_{1-p}$ and $\widehat{x}_{s}$ by first setting $m=\widehat{m}$ and by successively solving a single non-linear equation in the unknown probability mass $q$ (see, $(5))$ to the left of the mode $m$.

\section{SOLVING FOR THE PARAMETERS OF A TSP DISTRIBUTION}

Here we shall consider a slightly more general set-up by introducing an unrestricted quantile level $r$ in place of the pre-assigned $1-p$ for the upper quantile $b_{1-p}$. Thus, let $a_{p}$ and $b_{r}$ be the $p$-th and $r$ th quantiles, respectively, of the random variable $X$, such that 


$$
a<a_{p}<m<b_{r}<b .
$$

We propose elicitation of an additional quantile $x_{s}>m\left(x_{s}<m\right)$ when $m$ is smaller (larger) than the "midpoint" $\left(a_{p}+b_{r}\right) / 2$. Without loss of generality we shall consider the case

$$
a_{p}<m<x_{s}<b_{r}
$$

We are inclined to recommend the value 0.75 or 0.80 for $s$ taking into account that the more extreme values 0.10 and 0.90 have been suggested previously for $p$ and $r$, respectively, by Keefer and Verdini (1993). A possibly psychological advantage of the 80-th percentile over the 75-th one is that it is reminiscent of the $80 / 20$ rule popularized in economics (see, e.g., Barabasi 2003). Alternatively, a substantive expert could himself/herself specify the quantile level $s$, provided $p<s<r$.

From the $\operatorname{cdf}(3)$ and the definition of $a_{p}$ (i.e. $\left.F\left(a_{p} \mid a, m, b, n\right)=p\right)$ and the probability mass $q$ to the left of the mode $m(5)$ it now follows that

$$
a_{p}=a+\lambda(p, q, n)(m-a)
$$

where we have for the multiplier $\lambda(p, q, n)$ :

$$
0<\lambda(p, q, n)=(p / q)^{1 / n}<1 .
$$

Solving for $a$ from (10), and taking (9) into account, we have for given $n>0$

$$
a \equiv a(q \mid n)=a_{p}-\frac{\lambda(p, q, n)}{1-\lambda(p, q, n)}\left(m-a_{p}\right)<a_{p}
$$

(The notation $a(q \mid n)$ instead of $a$ is used here to indicate that the lower bound $a$ is a function of $q$ given $n$.) Analogously to (11) and (12), we have for $m<b_{r}$ (using the notation $b(q \mid n)$ in place of b):

$$
b \equiv b(q \mid n)=b_{r}+\frac{\mu(r, q, n)}{1-\mu(r, q, n)}\left(b_{r}-m\right)>b_{r} .
$$

where:

$$
0<\mu(r, q, n)=\{(1-r) /(1-q)\}^{1 / n}<1 .
$$


Substituting $a(q \mid n)$ and $b(q \mid n)$ as given by (12) and (13) into (5), we arrive at the following nonlinear equation

$$
\Phi(q \mid n)=q
$$

where $\Phi(q \mid n)$ involves $a_{p}, m, b_{r}, p$ and $r$ (that are pre-specified) and is given by

$$
\begin{aligned}
\Phi(q \mid n) & =\frac{m-a(q \mid n)}{b(q \mid n)-a(q \mid n)} \\
& =\frac{\left(m-a_{p}\right)\{1-\mu(r, q, n)\}}{\{1-\lambda(p, q, n)\}\left(b_{r}-m\right)+\{1-\mu(r, q, n)\}\left(m-a_{p}\right)},
\end{aligned}
$$

where $\lambda(p, q, n)$ and $\mu(r, q, n)$ are as above. In the Appendix, we shall show that, for a given value of $n>0$, one can numerically solve for the unique value of $q \in[p, r]$ using (15) and the explicit definition of $\Phi(q \mid n)$ (16) by means of, e.g., a bisection method (see Press et al. 1989) with the starting interval $[p, r]$. Hence, relations $(15)-(16)$ define a continuous implicit function $q(n)$ with domain $n>0$.

Next, we are able to calculate the lower bound $a^{\bullet}=a\{q(n) \mid n\}[(12)]$ and upper bound $b^{\bullet}=b\{q(n) \mid n\}[(13)]$ of a $\operatorname{TSP}\left[a^{\bullet}, m, b^{\bullet}, n\right]$ distribution given by the cdf

$$
\begin{gathered}
F_{X}\left(x \mid a^{\bullet}, m, b^{\bullet}, n\right)= \begin{cases}\frac{m-a^{*}}{b^{*}-a^{*}}\left(1-\frac{m-x}{m-a^{*}}\right)^{n} & a^{\bullet} \leq x \leq m \\
1-\frac{b^{*}-m}{b^{*}-a^{*}}\left(1+\frac{m-x}{b^{*}-m}\right)^{n} & m \leq x \leq b^{\bullet},\end{cases} \\
a^{\bullet}=a\{q(n) \mid n\}, b^{\bullet}=b\{q(n) \mid n\} .
\end{gathered}
$$

satisfying $a^{\bullet}<a_{p}<m<b_{r}<b^{\bullet}$ and $n>0$. [Compare (17) with (3).] We are introducing the notation $a^{\bullet}$ and $b^{\bullet}$ in (17) to distinguish them from (12) and (13) in which $q$ is not necessarily a function of $n$. Utilizing the continuities of $q(n), a\{q(n) \mid n\}$ and $b\{q(n) \mid n\}$ as functions of $n$ one can show that the $\operatorname{cdf}(17)$ converges as $n \downarrow 0$ to the two-value Bernoulli distribution with the probability mass

$$
q(0)=\frac{m-a_{p}}{b_{r}-a_{p}}
$$


at $a_{p}$ and the probability mass $[1-q(0)]$ at $b_{r}$. Similarly, it follows that as $n \rightarrow \infty$, the cdf $(17)$ converges to a (novel) reparameterization of an asymmetric Laplace cdf with parameters $a_{p}, m$ and $b_{1-r}$

$$
F_{X}\left(x \mid a_{p}, m, b_{r}\right)= \begin{cases}q(\infty)\left\{\frac{p}{q(\infty)}\right\}^{\frac{m-x}{m-a_{p}}} & x \leq m \\ 1-\{1-q(\infty)\}\left\{\frac{1-r}{1-q(\infty)}\right\}^{\frac{x-m}{b_{r}-m}} & x>m\end{cases}
$$

where $q(\infty)$ is the unique solution in $[p, r]$ of the equation

$$
h(q)=q,
$$

and the function $h(q)$ is given by

$$
h(q)=\frac{\left(m-a_{p}\right) \ln \left(\frac{1-r}{1-q}\right)}{\left(b_{r}-m\right) \ln \left(\frac{p}{q}\right)+\left(m-a_{p}\right) \ln \left(\frac{1-r}{1-q}\right)} .
$$

Equation (20) may be solved for using a bisection method with starting interval $[p, r]$ for $q$. (A standard parameterization of the asymmetric Laplace distribution is given for example in Kotz et al. 2001, p. 137). For $n=1$, the cdf (17) is simplified similarly to (3) to a uniform distribution with parameters

$$
a=\frac{r a_{p}-p b_{r}}{r-p}, b=\frac{(1-p) b_{r}-(1-r) a_{p}}{(r-p)}
$$

Hence, we can render the support of the cdf in (17) to be arbitrarily large by letting $n \rightarrow \infty$ or reduce it to its minimal value $\left\{a_{p}, b_{r}\right\}$ by letting $n \downarrow 0$ in the case that $m$ may also be an anti mode, or, if not, its minimal value $[a, b]$, where $[a, b]$ is given by $(22)$. While the values of $n$ in the interval $(0,1)$ are not consistent with an elicited mode $m$, the wide range of the TSP family (from a Bernoulli distribution to an asymmetric Laplace one) demonstrates its flexibility.

Returning to the example in Figure 1 where the lower quantile $a_{0.10}=6.5$, upper quantile $b_{0.90}=10.5$ and the most likely value (or the mode) $m=7$ are specified, we plot in Figure 2 two boundary cdfs within the TSP family that satisfy these specifications. As follows from (22), the one with minimal support width is a uniform distribution with support $[6,11]$. The other boundary with 
an unbounded support is the asymmetric Laplace cdf $(19)$, where $q(\infty) \approx 0.244$ is, as stated above, the unique solution to Eqs. $(20)-(21)$. The hatched region in Figure 2 is the feasibility area specified by these two boundary cdfs for the additional quantile $\left(x_{s}, s\right)$ given the percentiles as above $\left(a_{0.10}=6.5, b_{0.90}=10.5\right)$ and the mode $m=7$. This feasibility area is partitioned into two areas, satisfying $x_{s}<m$ and $x_{s}>m$. Note that the sub-area with $x_{s}>m$ satisfying $(9)$ is by far the largest since in this case $m<\left(a_{0.10}+b_{0.90}\right) / 2$. This observation indeed supports the earlier recommended elicitation of $x_{s}>m$, since the elicitation of a consistent quantile $x_{s}<m$ (in this example) may be well beyond the capabilities of a substantive expert due to the smaller size of its associated sub-area.

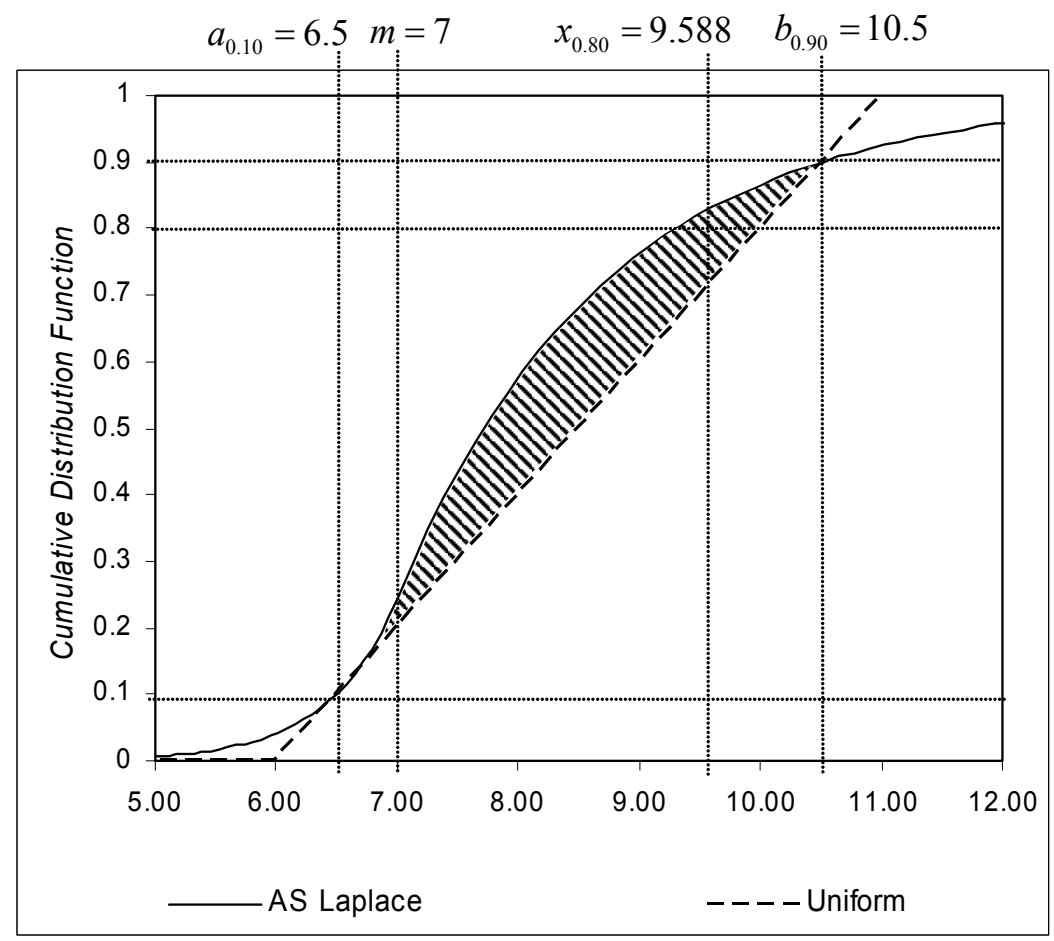

Figure 2. Feasibility region for the additional quantile $\left(x_{s}, s\right)$ satisfying $(9)$ given common percentiles $a_{0.10}=6.5, b_{0.90}=10.5$ and the mode $m=7$ specified by the two boundary CDFs. The first boundary is an asymmetric Laplace cdf (19) with $m=7$ and the second is an uniform cdf with bounds (22) $a=6, b=11$. 
If we now take, for example, the 80-th percentile of the beta distribution (1) with parameters

$$
\alpha=1.384, \beta=4.071, a=6, b=15
$$

plotted in Figure 1 (and thus satisfying $a_{0.10}=6.5, m=7$ and $b_{0.90}=10.5$ ) which is

$$
x_{0.80}=9.588
$$

it then follows immediately from Figure 2 that a $\operatorname{TSP}(a, m, b, n)$ distribution exists with these three quantiles $a_{0.10}, x_{0.80}, b_{0.90}$ and the mode $m=7$. Hence, as $a_{p}, x_{s}, b_{r}$ and $m$ are elicited from a substantive expert, a normative expert can immediately determine whether a member of the TSP family exists which is consistent with his/her assessment prior to solving for its parameters.

\subsection{Construction of the algorithm}

We are now in a position to formulate the algorithm to solve for the three remaining parameters $a, b$ and $n$ of a TSP distribution given a set of consistent quantiles $\left\{a_{p}, x_{s}, b_{r}\right\}$ and the mode $m$ satisfying (9). Obvious modifications can be made to the algorithm when $x_{s}<m$. The first four steps of the algorithm below determine an interval $\left[n_{\text {low }}, n_{\text {high }}\right]$ that contains the parameter solution $n^{*}$ of a TSP distribution with quantiles $\left\{a_{p}, x_{s}, b_{r}\right\}$ and the mode $m$ by starting with the uniform lower boundary cdf in Figure 2 (with $n=1)$. If $x_{s}$ is a quantile of the $\operatorname{TSP}\left(a^{\bullet}, m, b^{\bullet}, n\right)$ distribution in Step 2, it would follow that the $s^{\bullet}$ obtained in Step 2 equals $s$ and the algorithm terminates in Step 3. However, if the probability value $s^{\bullet}$ calculated in Step 2 is less than the quantile level $s$ of the additional quantile $x_{s}$, it follows that the $\operatorname{TSP}\left(a^{\bullet}, m, b^{\bullet}, n\right)$ cdf is too flat (see also Figure 2) and a larger value of $n$ is required leading to the adjustments of $n, n_{\text {low }}$ and $n_{\text {high }}$ in Step 4.

After having established the interval $\left[n_{\text {low }}, n_{\text {high }}\right]$, the remaining Steps 5-8 follow the bisection method by first setting $n^{\bullet}$ equal to the midpoint of this interval in Step 5. Steps 6 and 7 are analogous to Steps 2 and 3. If now the probability value $s^{\bullet}$ calculated in Step 6 is larger (less) than $s$ it follows that the $\operatorname{TSP}\left(a^{\bullet}, m, b^{\bullet}, n^{\bullet}\right)$ cdf is not flat enough (too flat) and the parameter solution $n^{*}$ 
for is contained in the interval $\left[n_{\text {low }}, n^{\bullet}\right]$ (the interval $\left[n^{\bullet}, n_{\text {high }}\right]$ ) suggesting adjustments of $n_{\text {low }}$ and $n_{h i g h}$ in Step 8. Explicitly the eight steps are:

Step 1 : Set $n=1$.

Step 2 : Solve for $q(n)$ from (15) and (16) using the bisection method with the starting interval $[p, r]$ for $q$.

Calculate $a^{\bullet}=a\{q(n) \mid n\}$ from (12).

Calculate $b^{\bullet}=b\{q(n) \mid n\}$ from (13).

Calculate $s^{\bullet}=F_{X}\left(x_{s} \mid a^{\bullet}, m, b^{\bullet}, n\right)$.

Step 3 : If $\left|s-s^{\bullet}\right|<\epsilon$ then STOP.

Step $4:$ If $s^{\bullet}<s$ then

set $n=2 n, n_{\text {low }}=n, n_{\text {high }}=2 n$, Goto Step 2 .

Step 5 : Set $n^{\bullet}=\left(n_{\text {low }}+n_{\text {high }}\right) / 2$.

Step 6 : Solve for $q\left(n^{*}\right)$ from (15) and (16) using the bisection method with the starting interval $[p, r]$ for $q$.

Calculate $a^{\bullet}=a\left\{q\left(n^{\bullet}\right) \mid n^{\bullet}\right\}$ from (12).

Calculate $b^{\bullet}=b\left\{q\left(n^{\bullet}\right) \mid n^{\bullet}\right\}$ from (13).

Calculate $s^{\bullet}=F_{X}\left(x_{s} \mid a^{\bullet}, m, b^{\bullet}, n^{\bullet}\right)$.

Step 7 : If $\left|s-s^{\bullet}\right|<\epsilon$ then STOP.

Step $8:$ If $s^{\bullet}>s$ then

$$
n_{\text {high }}=n^{\bullet}
$$

else

$$
n_{\text {low }}=n^{\bullet} \text {. }
$$

Goto Step 5.

A software program with an implementation of the above algorithm is available from the authors upon request. 
Applying the algorithm above to the beta distribution $(23)$ where $a_{0.10}=6.5, x_{0.80}=0.9588$, $b_{0.90}=10.5$ and $m=7$ (as depicted in Figure 2) yields

$$
n^{*}=2.782, a^{*}=a\left\{q\left(n^{*}\right) \mid n^{*}\right\}=5.034, b^{*}=b\left\{q\left(n^{*}\right) \mid n^{*}\right\}=13.722 .
$$

Figure 3 plots the pdf and the cdf of the beta distribution (23) and that of the $\operatorname{TSP}\left(a^{*}, m, b^{*}, n^{*}\right)$ distribution with $m=7$. In Figure 3, the common percentiles (mode) of the beta and TSP cdfs are indicated by dotted lines. Table 2 compares these distributions in terms of their lower and upper bounds, means, variances, skewnesses and kurtoses.
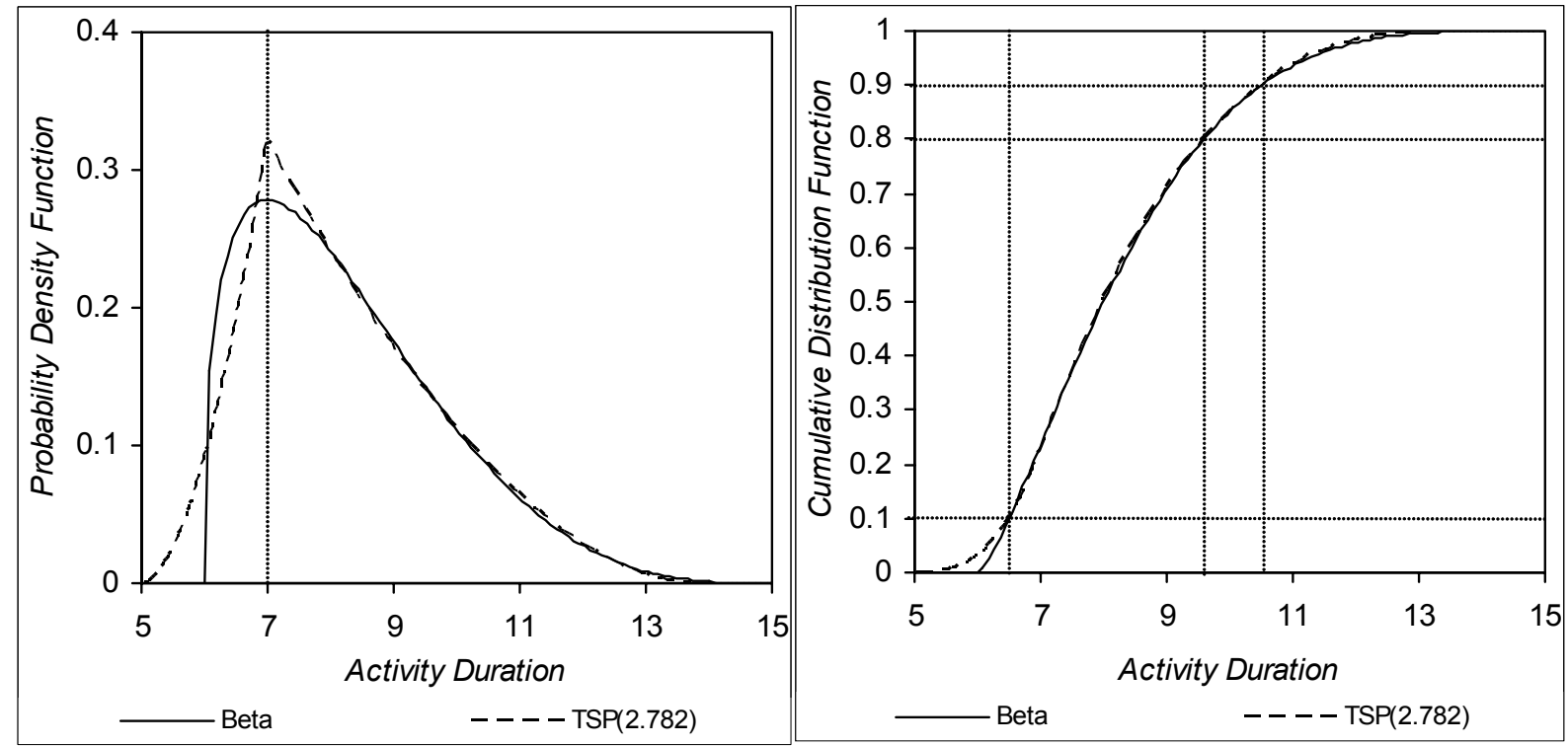

Figure 3. PDF and CDF of the beta distribution with parameters (23) and TSP distribution with parameters (25) (presented in the second row of Table 2) with the common percentiles $a_{0.10}=6.5, x_{0.80}=9.588, b_{0.90}=10.5$ and the most likely value (the mode) $m=7$.

Table 2. Summary statistics of a beta distribution with parameters (23) and a TSP distribution with parameters (25). Both distributions have common percentiles $a_{0.10}=6.5$,

$$
b_{0.90}=10.5, x_{0.80}=9.588 \text { and mode } m=7 .
$$

\begin{tabular}{ccccccc}
\hline & Lower Bound & Upper Bound & Mean & Variance & Skewness & Kurtosis \\
\hline Beta & 6.00 & 15.00 & 8.28 & 2.38 & 0.60 & 3.08 \\
TSP & 5.03 & 13.72 & 8.26 & 2.38 & 0.43 & 2.88 \\
\hline
\end{tabular}


Note that the cdfs in Figure 3 are almost indistinguishable in the range from $\left[a_{0.10}, b_{0.90}\right]$ and that the individual statistics in Table 2 align better than those for the four distributions in Table 1 presented in Section 2, since the distributions in Table 2 possess an additional quantile $x_{s}$ in common.

\subsection{Sensitivity of additional quantile specification}

For every specification of an additional quantile $x_{s}$ in the hatched region of Figure 2 a unique value of the parameter $n$ may be obtained by the algorithm above. Each value for the parameter $n$ results in turn, via $a\{q(n) \mid n\}(12)$ and $b\{q(n) \mid n\}$ (13), in a TSP distribution defined by Eq. (17). Hence, the hatched region in Figure 2, may also be interpreted as a sensitivity region for the additional quantile specification as it defines the coverage area of all TSP cdfs with common lower quantile $a_{0.10}=6.5$, upper quantile $b_{0.90}=10.5$ and mode $m=7$.

We may further investigate the sensitivity of the mean and variance with regard to the additional quantile specification (keeping $a_{0.10}=6.5, b_{0.90}=10.5$ and mode $m=7$ fixed) in the algorithm above, by studying the behavior of the coefficient of variation of the distribution (17). Figure 4 plots the coefficient of variation $C V[X]$ (i.e. $\{$ St. Dev. $/ E[X]\} \times 100 \%$ ) as a function of $n$ (which may easily be evaluated by substituting the value for $a\{q(n) \mid n\}[(12)]$ and upper bound $b\{q(n) \mid n\}[(13)]$, obtained from the algorithm, in equation (6)). We observe here a larger sensitivity at the lower ranges of the parameter $n$ (i.e. closer to the lower boundary of the hatched region in Figure 2). The coefficient of variations for the TSP distributions in Figure 1 and Figure 3 are indicated separately.

It is appropriate to point out here that specification of a lower quantile $a_{p}$, a most like value $m$, an upper quantile $b_{r}$, and one additional quantile $x_{s}$ of a bounded uncertain phenomenon does not determine the family of distributions to be fitted. However, we are not aware of an algorithm as described above, including $x_{s}$ boundary conditions, for the beta distribution (nor for any other continuous univariate bounded distribution). While the algorithm does fit "exactly" (i.e. up to a desirable accuracy level) a TSP distribution to the provided quantiles and most likely value (if 
mutually consistent), it evidently does not account for potential inaccuracy of the experts assessments. Sensitivity with respect to additional quantile specification is indicated in Figure 4 for our example with $a_{0.10}=6.5, b_{0.90}=10.5$ and most likely value $m=7$. We would expect similar or larger CV sensitivity with respect to the specification of the lower and upper quantiles and most likely value (keeping the other three fixed) and plan to address this in a future paper.

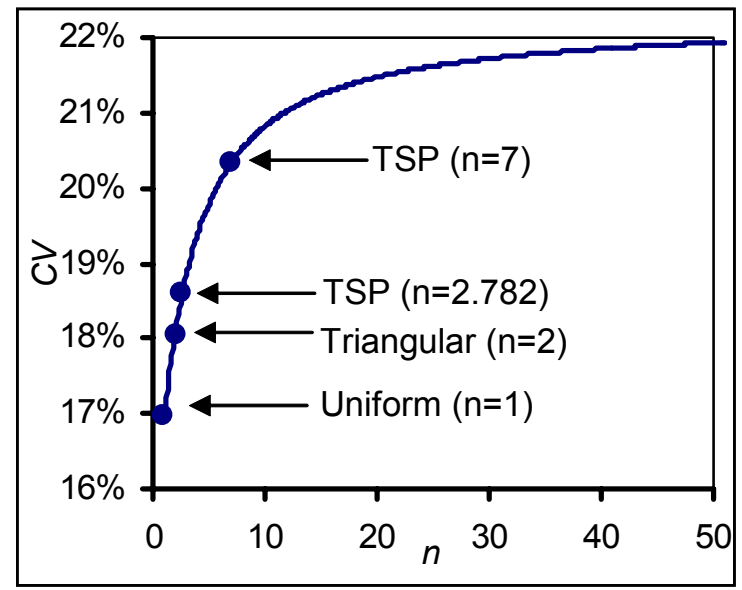

Figure 4. Sensitivity of additional quantile $x_{s}$ specification in terms of the coefficient of variation, keeping $a_{0.10}=6.5, b_{0.90}=10.5$ and most likely value $m=7$ fixed.

\section{EXAMPLES}

We shall now present two examples to illustrate the effect of specifying an additional quantile $x_{s}$, after already elicited lower and upper quantiles $a_{p}$ and $b_{r}$ and the most likely value $m$, in the course of specifying the input distributions with bounded support in simulations in the absence of data.

\subsection{A $M / G / 1$ queuing system}

Consider an $\mathrm{M} / \mathrm{G} / 1$ queuing system with inter-arrival times exponentially distributed with the mean of 8.5/0.95 $\approx 8.95$ minutes (8.5 is the average of the uniform distribution in Figure 1 and Table 1) and service times distributed according to one of the distributions depicted in Figures 1 and 3. From the means of these service times distributions provided in Table 1, it thus follows that the associated 
service utilizations range from 0.95 (the uniform case) to approximately $8.95 / 8.20 \approx 0.92$ (the TSP(7) case). We may simulate customer waiting times by sampling from the service time distributions and inter-arrival distributions and by applying Lindley's (1952) recursion

$$
D_{i+1}=\max \left\{0, D_{i}+S_{i}-A_{i+1}\right\}, i \geq 1
$$

where $A_{i}$ is the sequence of inter-arrival times, $S_{i}$ is the sequence of service times, and $D_{i}$ is the sequence of customer waiting times. Since we start with an empty system, we use the method of Welch (1983) to remove the initialization bias. This procedure allows us to visually estimate a threshold $l$ beyond which we may assume that our M/G/1 simulation has reached its stationary state. (See, e.g., Alexopoulos and Seila (1998) for a more detailed description of the procedure.) Since the uniform distribution in Table 1 has the highest mean service time, we shall determine $l$ for this $\mathrm{M} / \mathrm{G} / 1$ setup and apply the same initialization threshold $l$ to the other service time distributions as well.

Figure 5 consists of three parts. Figure 5A depicts the waiting time occurrences $Y_{1 j}$ of customer $j$ in Replication 1 for $j=1, \ldots, 20000$. Figure 5B plots the average waiting time $\bar{Y}_{j}$ of customer $j$ over 50 of such replications of the same lengths, i.e.

$$
\bar{Y}_{j}=\sum_{i=1}^{50} Y_{i j}, j=1, \ldots, 20000 .
$$

Finally, Figure 5C graphs the moving average

$$
\bar{Y}_{j}(w)= \begin{cases}\frac{1}{2 w+1} \sum_{m=-w}^{w} \bar{Y}_{j} & w+1 \leq j \leq 20000-w \\ \frac{1}{2 j-1} \sum_{m=-j+1}^{j-1} \bar{Y}_{j} \quad 1 \leq j \leq w\end{cases}
$$

with a time window $w=3000$ (indicated in Figure 5B) sufficiently large to include multiple regeneration cycles and less than $\lfloor 2000 / 4\rfloor$ as suggested by Law and Kelton (2000). Noting that an $\mathrm{M} / \mathrm{G} / 1$ system regenerates itself each time an arriving customer does not have to wait, one may observe typical regeneration epochs in Figure 5A. 

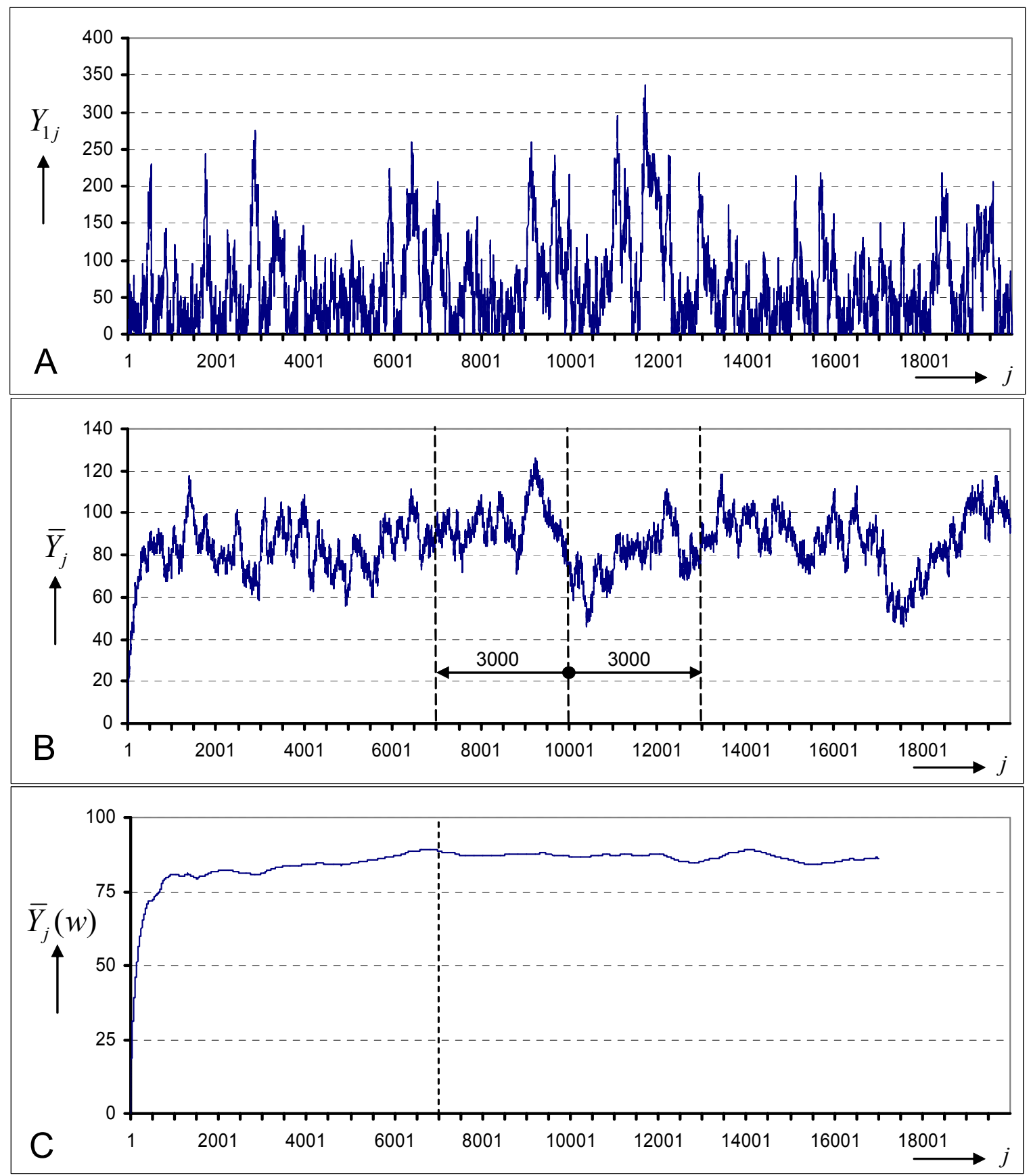

Figure 5. Welch (1983) analysis for an M/G/1 simulation with mean inter-arrival time of $\approx 8.95$ minutes and an uniform $[6,11]$ service time distribution.

From Figure 5C we observe an upward trend of the moving average $\bar{Y}_{j}(w)$ up to approximately customer $l=7000$. Hence, to estimate stationary waiting time distributions for the five difference 
service time distributions in Figures 1 and 3, we shall collect waiting times after $l=7000$. Figure 6 presents the stationary waiting time cdfs constructed from the waiting times $Y_{i j}, i=1, \ldots 5$, $j=7001, \ldots, 20000$, of five additional replications resulting in a sample size of 65000 for each of these service time distributions. In generating Figure 6 we have applied the common random numbers technique (CRN) (see, e.g. Banks et al. 2005). That is, each replication has a specified sequence of independent uniform $[0,1]$ pseudo-random numbers for its arrival time generation and a separate one for its service time generation. In addition, the sequences of pseudo-random numbers across these five replications are sampled independently from each other. However, the same random numbers in these five replications are used when changing the service time distribution from the uniform one to any of the other four service time distributions in Figures 1 and 3. Note that the estimated waiting time cdf corresponding to the triangular service distribution is displayed in Figures $6 \mathrm{~A}$ and $\mathrm{B}$.

From Figure 6A one observes that the vertical distances between the cdfs in this figure are quite large. While the maximal difference of the probability that a customer does not have to wait amongst these waiting time cdfs equals approximately 0.03 (which is consistent with the difference between service utilizations mentioned in the first paragraph of this Section), the maximal vertical distance between the cdfs equals approximately 0.214 at the waiting time of 78.2 minutes. Recall that all five distributions in Figures 1 and 3 have the same lower and upper quantiles $\left(a_{0.10}=6.5, b_{0.90}=10.5\right)$ and the most likely value $(m=7)$. Hence, the differences amongst the cdfs in Figure 6A arise solely from different values for the shape parameter $n$ for the fitted TSP service distributions.

Consequently, a normative expert may be quite uncomfortable with these results, since a particular choice for the value of the parameter $n$ could be considered arbitrary (including the value $n=2$ corresponding to the triangular distribution). Thus our suggestion in Section 2 to elicit an additional quantile $x_{s}$ to determine the value of the shape parameter $n$ makes sense. 


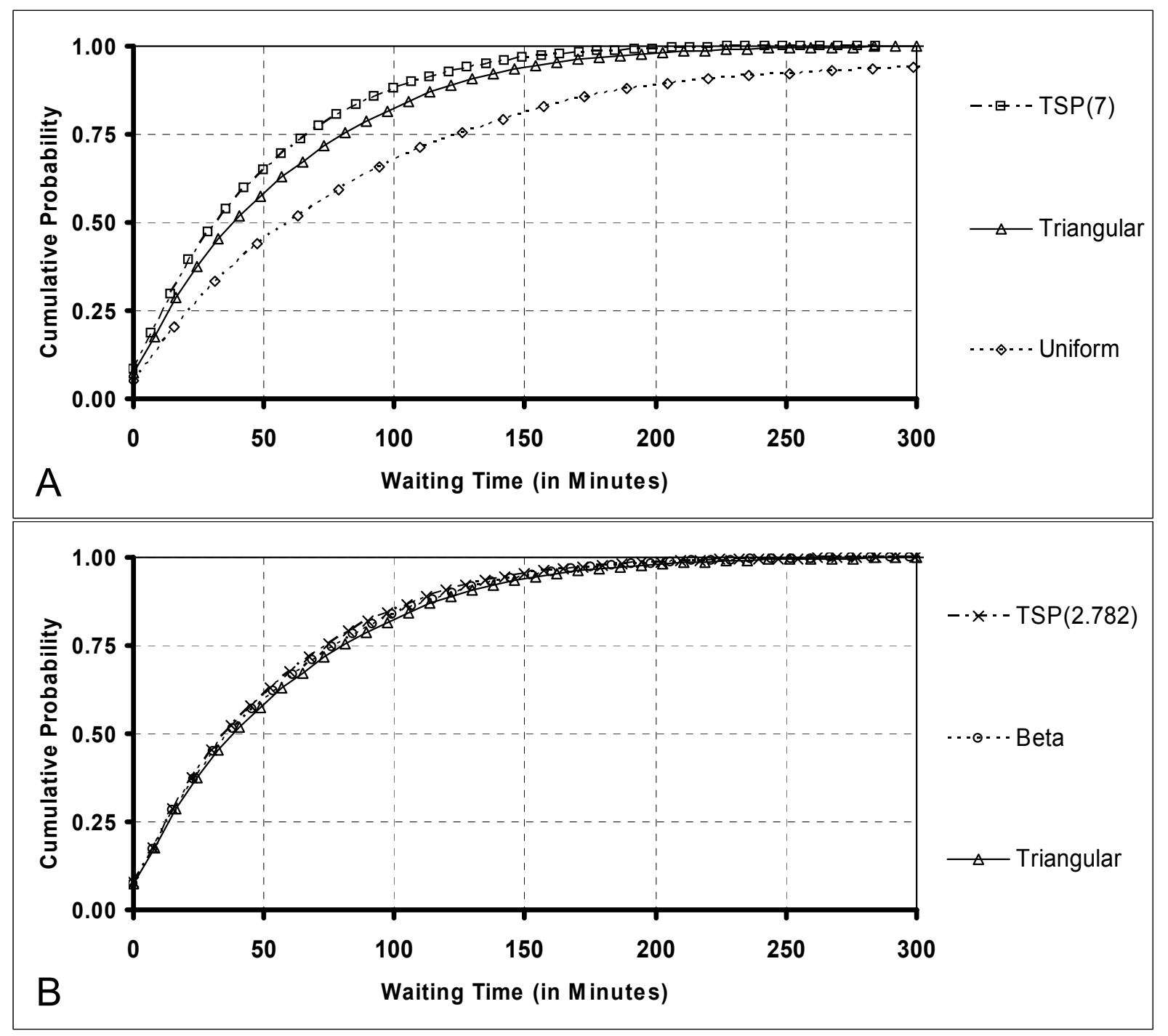

Figure 6. Estimated stationary waiting time distributions employing 65000 samples for the M/G/1 queuing simulation with mean inter-arrival time of $\approx 8.95$ minutes and service time distributions depicted in Figures 1 and 3.

Figure 6B displays the waiting time cdfs employing as service distributions the cdfs in Figure 3 and the triangular one in Figure 1. The TSP(2.782) and beta service distributions involve besides the values $a_{0.10}, m$ and $b_{0.90}$ a common additional quantile $x_{0.80}$. The maximal distance between the corresponding waiting time cdfs in Figure 6B is approximately just 0.018 at the waiting time of 67.4 minutes (being about 1/12-th of the difference observed in Figure 6A). Moreover, the maximal 
difference between the waiting time cdfs associated with the beta and triangular service distributions (sharing only $a_{0.10}, m$ and $b_{0.90}$ ) in Figure $6 \mathrm{~B}$ is comparatively small as well and constitutes approximately 0.014 at a somewhat higher waiting time of 68.7 minutes. Hence, it seems that a normative expert who is comfortable limiting himself/herself to beta and triangular family of distributions for modeling bounded input phenomena in simulations in the absence of data will arrive at similar output analysis results also in the case when the beta and triangular distributions share lower $a_{0.10}$ and upper quantiles $b_{0.90}$ and the most likely value $m$. Given these quantiles and the most likely value, the lower bound $a$ and upper bound $b$ of the triangular distribution may be easily determined from Eqs. (11) - (16) using a bisection method to solve equation (15). To the best of our knowledge, no algorithm is available that solves for the lower and upper bounds of a beta distribution given these quantiles and a most likely value. We shall further amplify the above observations via a PERT example in the next sub-section.

\subsection{A project network example}

We shall cast the results of this paper in a chronological context of what we call a 40 year PERT "controversy" (see, e.g., Clark 1962, Grubbs 1962, Moder and Rogers 1968, Elmaghraby 1978, Keefer and Verdini 1993, Kamburowski 1997, Johnson 1997, Lau et al. 1998, and Herrerías et al. 2003, among others) regarding the estimation of the parameters $\alpha$ and $\beta$ of the beta distribution (1) using the formulas

$$
\begin{aligned}
& E[T]=\frac{a+4 m+b}{6} \\
& \operatorname{Var}[T]=\frac{1}{36}(b-a)^{2},
\end{aligned}
$$

(as suggested originally by Malcolm et al. 1959) $)^{3}$. Here, $T$ is a random variable modeling an activities completion time, $a$ and $b$ are lower and upper bound estimates and $m$ is a most like estimate for $T$.

\footnotetext{
${ }^{3}$ Kamburowski (1997) in a recent publication notes that: "Despite the criticisms and the abundance of new estimates, the PERT mean and variance [given by (29) in this paper] can be found in almost every textbook on OR/MS and P/OM, and are employed in much project management software."
} 
The remaining beta parameters $\alpha$ and $\beta$ in (1) are obtained from (29) utilizing the method of moments. We shall compare six different scenarios, described in Table 3, for calculating the completion time distribution of the project network in Figure 7 employing the lower and upper bounds $a$ and $b$ and most likely value $m$ provided in Table 4. While both Scenarios 2 and 4 involve triangular distributions, Scenario 4 in Table 3 is designated Triangular to coordinate with the example in Section 4.1; Scenario 2 is designated equivalently as $\operatorname{TSP}(2)$. The network in Figure 7 is a small 18-activity project network and adapted from ship-building applications (Taggart 1980).

Table 3. Scenarios for the completion time distribution calculations of the project in Figure 7

\begin{tabular}{|c|c|}
\hline $\begin{array}{l}\text { Scenario 1: } \\
\text { Beta }\end{array}$ & $\begin{array}{l}\text { Activity durations uncertainties will be modeled via beta distributions using the pessimistic } \\
a \text {, most likely } m \text { and the optimistic estimates } n \text { specified in Table } 4 \text { using formulas }(29) \text {. } \\
\text { Table } 4 \text { provides the values for the parameters } \alpha \text { and } \beta \text { of the beta distributions which } \\
\text { follow from (29) using the methods of moments. }\end{array}$ \\
\hline $\begin{array}{l}\text { Scenario 2: } \\
\operatorname{TSP}(2)\end{array}$ & $\begin{array}{l}\text { Activity durations uncertainties will be modeled via triangular distributions using the } \\
\text { pessimistic } a \text {, most likely } m \text { and the optimistic } b \text { estimates specified in Table } 4 \text {. }\end{array}$ \\
\hline $\begin{array}{l}\text { Scenario 3: } \\
\text { Uniform }\end{array}$ & $\begin{array}{l}\text { We solve for the lower } a_{0.10} \text { and upper quantiles } b_{0.90} \text { of the Scenario } 1 \text { beta distributions. } \\
\text { Their values are provided in Table } 4 \text {. Next, activity durations uncertainties will be modeled } \\
\text { via uniform distributions fitted to these quantiles by calculating their boundary parameters } \\
\text { using equation }(22) \text {. }\end{array}$ \\
\hline $\begin{array}{l}\text { Scenario 4: } \\
\text { Triangular }\end{array}$ & $\begin{array}{l}\text { We solve for the lower } a_{0.10} \text { and upper } b_{0.90} \text { quantiles, and most likely value } m^{*} \text { of the } \\
\text { Scenario } 1 \text { beta distributions. Their values are provided in Table } 4 \text {. Next, activity durations } \\
\text { uncertainties will be modeled via triangular distributions fitted to these quantiles and most } \\
\text { likely value. Namely, their lower and upper bounds will be calculated by first setting } n=2 \text {, } \\
\text { next by solving for } q(2) \text { using equations }(15) \text { and }(16) \text { and finally by substituting the value } \\
\text { of } q(2) \text { in equations }(12) \text { and }(13) \text {. }\end{array}$ \\
\hline $\begin{array}{l}\text { Scenario 5: } \\
\operatorname{TSP}(7)\end{array}$ & $\begin{array}{l}\text { Same as Scenario 4, but activity durations uncertainties will be modeled via TSP distributions } \\
\text { with } n=7 \text { instead of } n=2 \text {. }\end{array}$ \\
\hline $\begin{array}{l}\text { Scenario 6: } \\
\operatorname{TSP}(n)\end{array}$ & $\begin{array}{l}\text { We solve for the lower } a_{0.10} \text { and upper quantiles } b_{0.90} \text {, most likely value } m^{*} \text { and additional } \\
\text { quantile } x_{0.80} \text { of the Scenario } 1 \text { beta distributions. Their values are provided in Table } 4 \text {. } \\
\text { Next, activity durations uncertainties will be modeled via TSP distributions fitted to these } \\
\text { quantiles and most likely value using the algorithm in Section } 3.1 \text {. }\end{array}$ \\
\hline
\end{tabular}

We now generate the cdf of the completion time distribution of the project presented in Figure 7 for each one of the six scenarios in Table 3 employing the Monte Carlo technique (Vose 1996) involving 25000 samples from the activity durations and subsequently applying the critical path method (CPM) (Winston 1993). Consequently, for each scenario we shall obtain an output sample 


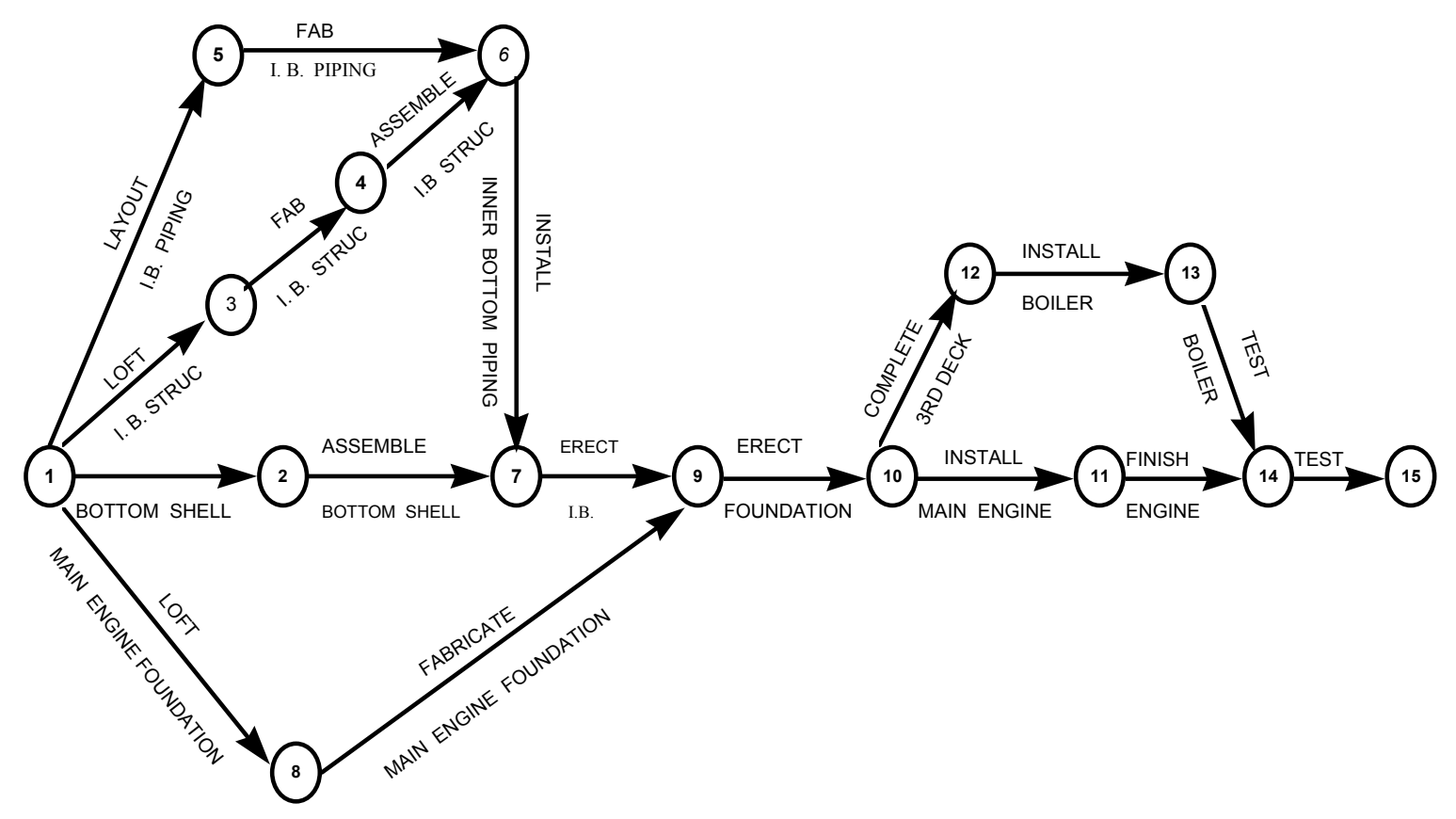

Figure 7. Example project network $\mathcal{P}$ for a production process.

Table 4. Technical data for modeling the uncertainty in activity durations for the project network in Figure 7 and for Scenarios 1-6 in Table 3.

\begin{tabular}{lrrrrrrrrrrr}
\hline Activity Name & $a$ & $m$ & $b$ & $E[T]$ & $\operatorname{Var}[T]$ & $\alpha$ & $\beta$ & $a_{0.10}$ & $m^{*}$ & $x_{0.80}$ & $b_{0.90}$ \\
\hline Shell:Loft & 22 & 25 & 31 & 25.5 & 2.3 & 2.9 & 4.6 & 23.6 & 25.1 & 26.8 & 27.6 \\
Shell: Assemble & 35 & 38 & 43 & 38.3 & 1.8 & 3.2 & 4.5 & 36.6 & 38.1 & 39.5 & 40.1 \\
I.B.Piping: Layout & 19 & 22 & 47 & 25.7 & 21.8 & 1.3 & 4.2 & 20.4 & 21.5 & 29.6 & 32.4 \\
I.B.Piping: Fab. & 6 & 7 & 15 & 8.2 & 2.3 & 1.3 & 4.2 & 6.5 & 6.9 & 9.4 & 10.3 \\
I.B. Structure: Layout & 23 & 24 & 30 & 24.8 & 1.4 & 1.6 & 4.4 & 23.5 & 24.0 & 25.8 & 26.5 \\
I.B. Structure: Fab. & 14 & 18 & 24 & 18.3 & 2.8 & 3.4 & 4.4 & 16.2 & 18.1 & 19.8 & 20.6 \\
I.B. Structure: Assemb. & 9 & 14 & 20 & 14.2 & 3.4 & 3.7 & 4.2 & 11.8 & 14.1 & 15.8 & 16.6 \\
I.B.Structure: Install & 5 & 7 & 13 & 7.7 & 1.8 & 2.3 & 4.7 & 6.0 & 7.1 & 8.8 & 9.5 \\
Mach. Fdn. Loft & 26 & 28 & 33 & 28.5 & 1.4 & 2.6 & 4.7 & 27.0 & 28.1 & 29.5 & 30.1 \\
Mach. Fdn. Fabricate & 20 & 35 & 51 & 35.2 & 26.7 & 3.9 & 4.1 & 28.3 & 35.1 & 39.8 & 42.1 \\
Erect I.B. & 27 & 30 & 37 & 30.7 & 2.8 & 2.7 & 4.7 & 28.6 & 30.2 & 32.1 & 33.0 \\
Erect Foundation & 5 & 7 & 14 & 7.8 & 2.3 & 2.1 & 4.6 & 6.0 & 7.1 & 9.1 & 9.9 \\
Complete 3rd Deck & 4 & 5 & 9 & 5.5 & 0.7 & 2.0 & 4.6 & 4.5 & 5.1 & 6.2 & 6.7 \\
Boiler: Install & 6 & 7 & 12 & 7.7 & 1.0 & 1.7 & 4.5 & 6.5 & 7.0 & 8.5 & 9.1 \\
Boiler: Test & 9 & 10 & 16 & 10.8 & 1.4 & 1.6 & 4.4 & 9.5 & 10.0 & 11.8 & 12.5 \\
Engine: Install & 6 & 7 & 15 & 8.2 & 2.3 & 1.3 & 4.2 & 6.5 & 6.9 & 9.4 & 10.3 \\
Engine: Finish & 19 & 20 & 26 & 20.8 & 1.4 & 1.6 & 4.4 & 19.5 & 20.0 & 21.8 & 22.5 \\
Final Test & 13 & 15 & 24 & 16.2 & 3.4 & 1.8 & 4.5 & 14.0 & 15.1 & 17.7 & 18.8 \\
\hline
\end{tabular}


of size 25000 for the completion time of the project network in Figure 7 (from which one then empirically estimates its completion time distribution). The resulting cdfs for the six scenarios described above are depicted in Figures 8A - C.

The comparison between "Scenario 1: Beta" and "Scenario 2: TSP(2)" in Figure 8A may explain (albeit partially) the reason that the use of (29) is quite controversial. Indeed, the parameters of the beta and triangular distributions in these scenarios are "estimated" from the same values of $a, m$ and $b$ (in Table 4). However, the maximal vertical distance between the two cdfs approaches to over 0.50 at 153 days. This difference could be used as a benchmark for the maximal vertical differences observed in Figures 8B and 8C.

The maximal vertical distance between the three cdfs depicted in Figure 8B equals approximately 0.13 at 149 days (constituting about one-fourth of the difference in Figure 8A). Recall that the identical activity times utilized to generate Figure $8 \mathrm{~B}$ all have the same lower and upper quantiles $a_{0.10}$ and $b_{0.90}$ and the most likely value $m^{*}$ (presented in Table 4). The differences between the cdfs in Figure 8B are thus being the result of using the different values 1,2 and 7 for the shape parameter $n$ of the TSP distributions for "Scenarios 3: Uniform", "Scenario 4: Triangular" and "Scenario 5: TSP(7)", respectively. In light of the prolonged controversy regarding the use of (29) (resulting in a substantial difference between cdfs observed in Figure 8A), we are of a strong opinion that a normative expert should inevitably also be uncomfortable with choosing a particular value for $n$ (resulting in a difference between cdfs observed in Figure 8B). In fact, any choice for the value of the shape parameter $n$, in the absence of data, when a lower and upper quantiles $a_{p}$ and $b_{r}$ respectively and the most likely value $m$ are given, is arbitrary (not unlike the specification of $E[T]$ and $\operatorname{Var}[T]$ in $(29))$. Hence, our suggestion in Section 2 to elicit an additional quantile $x_{s}$ from the substantive expert is appropriate. Furthermore, the algorithm in Section 3.1 allows a normative expert to solve for the shape parameter $n$. The additional quantiles $x_{0.80}$ in this example are obtained from the beta distributions associated with Scenario 1 in Table 3 and are listed in Table 4. Scenario 6 in Table 6 utilizes these quantiles to solve for the parameters $n$ for each and every activity duration. The maximal vertical difference between the "Scenario 1: Beta" and "Scenario 6: TSP $(n)$ " 

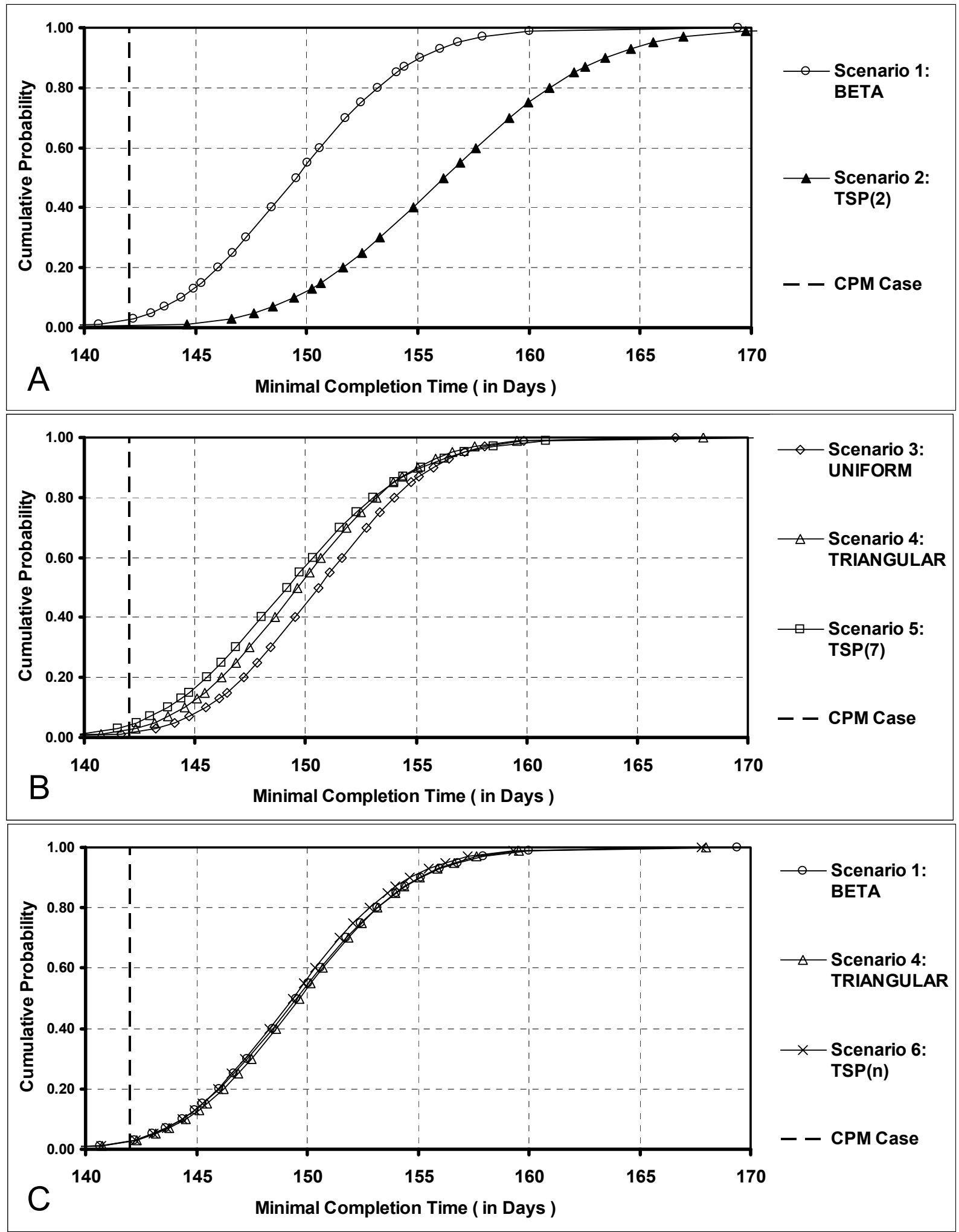

Figure 8. Comparison of cdfs of the completion times for the project sketched in Figure 7 for the six scenarios described in Table 3. 
cdfs in Figure 8C now equals approximately 0.02 obtained at 153 days (which is barely $1 / 25$-th of the difference observed in Figure 8A and just about one-sixth of the difference in Figure 8B).

For completeness in Figure 8C a comparison between the "Scenario 1:Beta" and "Scenario 4: Triangular" cdfs should be noted. Here the maximal vertical difference also turns out to be 0.02 at 148 days. Hence, for those normative experts that are comfortable to confine themselves to the beta or triangular families in the course of modeling input distributions with bounded support in the absence of data, the graphs in Figure 8C (and Figure 5B) seem to indicate that when the coinciding beta and triangular distributions have common lower and upper quantiles $a_{0.10}$ and $b_{0.90}$ and most likely value $m$, quite similar output results are to be expected. We stress that our opinion is that a normative expert should not be satisfied with such a restriction in view of the potential differences that are indicated in Figure 8B.

Note that in Figure 8 the project completion time of 142 days following from the CPM method (using only the most likely values of $m$ in Table 4) is represented by the bold vertical line. Since the values of the mode $m$ are less than the midpoint $(a+b) / 2$ for all the 18 activities in Table 4 (which allows the execution of the algorithm in the form presented in Section 3.1), we deduce from Figure 8 that the probability of achieving the completion time of 142 days is less than 0.05 for all the six scenarios. Granted that the skewness of the activity distributions in Table 3 may perhaps be somewhat inflated, the case could definitely be made that the skewness towards the lower bound appears in the assessed activity time distributions due to a motivational bias of the substantive expert. Moreover, the probability being less than 0.05 of attaining a 142 day deadline for the project sketched in Figure 7 reinforces the well known rule that, in applications, uncertainty results ought to be conveyed to the decision makers.

\section{A CONCLUDING REMARK}

The examples in the previous section fall short of a rigorous proof that assures us that the only family which can be fitted to the estimated lower and upper quantiles $a_{p}$ and $b_{r}$, the most likely value $m$ and an additional quantile $x_{s}$, with $a_{p}<x_{s}<b_{r}$, is the TSP family. However, the conclusions 
stemming from both examples do provide us with a further justification for eliciting an additional quantile, supplementing lower and upper quantiles and a most likely value, in the course of specifying input distributions with bounded support for simulations via expert judgment. We are confident that we have simplified the dilemma of a normative expert regarding the use of a particular family of distributions for a bounded uncertain phenomenon in the absence of data, by providing a flexible alternative to the beta distribution (1) employing instead the TSP distribution (2) that is motivated by an intuitive appeal of the triangular distribution (a member of the TSP family). The TSP distribution allows for computationally efficient sampling and permits us to devise a straightforward algorithm to solve for the distributional parameters based on the well grounded stipulation that the actual lower and upper bounds per se, in a majority of cases, are quite cumbersome to assess by substantive experts.

\section{ACKNOWLEDGEMENT}

The authors appreciate the useful discussions with our colleagues Hernan Abeledo, Enrique Campos-Nanez, Thomas Mazzuchi in the course of preparation of this paper. Last but certainly not least, we are thankful to the referees and the editor of the Simulation Department of the IIE Transactions whose devotion far beyond the call of duty and valuable comments improved substantially the contents and presentation of earlier versions.

\section{APPENDIX. THE IMPLICIT FUNCTION $q(n)$}

In this Appendix we shall elaborate on the implicit function $q(n)$, which seems to be a new concept introduced in this paper. This function plays a pivotal role in the algorithm described in Section 3.

Let $X \sim T S P(a, m, b, n)$ with pdf (2) and cdf (3). From inequality (9) and the probability mass $q(5)$ to the left of the mode (the most likely value) $m$ we obtain the relations

$$
0<p<q<r<1 \Rightarrow 0<\frac{p}{q}<1 \text { and } 0<\frac{1-r}{1-q}<1 .
$$


where $p$ and $r$ are the quantile levels of the quantiles $a_{p}$ and $b_{r}$, respectively. Taking partial derivatives with respect to $n$ and $q$ of the function $a(q \mid n)$ defined in (12) we arrive at

$$
\frac{\partial}{\partial n} a(q \mid n)=\ln \left(\frac{p}{q}\right) \frac{\lambda(p, q, n)}{\{1-\lambda(p, q, n)\}^{2}} \frac{m-a_{p}}{n^{2}}<0
$$

and

$$
\frac{\partial a(q \mid n)}{\partial q}=\frac{\lambda(p, q, n)}{\{1-\lambda(p, q, n)\}^{2}} \frac{m-a_{p}}{n q}>0,
$$

where $\lambda(p, q, n)$ is defined by $(11)$. Hence, the lower bound $a(q \mid n)$ is a strictly decreasing (increasing) function of $n$ (of $q$ ). In addition, from (9), (11) and (30) it follows that

$$
a(q \mid n) \rightarrow-\infty \text { as } n \rightarrow \infty \quad(q \downarrow p)
$$

for the values of $q \in(p, r)$ (of $n>0$ ). Analogously, taking partial derivatives with respect to $n$ and $q$ of the function $b(q \mid n)(13)$ we arrive at

$$
\frac{\partial b(q \mid n)}{\partial n}=-\ln \left(\frac{1-r}{1-q}\right) \frac{\mu(r, q, n)}{\{1-\mu(r, q, n)\}^{2}} \frac{b_{r}-m}{n^{2}}>0,
$$

and

$$
\frac{\partial b(q \mid n)}{\partial q}=\frac{\mu(r, q, n)}{\{1-\mu(r, q, n)\}^{2}} \frac{b_{r}-m}{n(1-q)}>0,
$$

where $\mu(r, q, n)$ is defined by (14). Hence, the upper bound $b(q \mid n)$ is a strictly increasing function of both $n$ and $q$. From (9), (14) and (30) it follows that

$$
b(q \mid n) \rightarrow \infty \text { as } n \rightarrow \infty \quad(q \uparrow r)
$$

for $q \in(p, r)($ of $n>0)$.

The Basic Lemma : Equation $(15), \Phi(q \mid n)=q$, has a unique solution $q(n) \in(p, r)$ for every fixed value $n>0$, where the function $\Phi(q \mid n)$ is defined in (16), and the resulting implicit function $q(n)$ is continuous. Proof: Let $n>0$ be fixed. Substituting $q=p$ into (16) we have

$$
\Phi(p \mid n)=1,
$$


and analogously substituting $q=r$ into (16) we have

$$
\Phi(r \mid n)=0 .
$$

From the continuity of (16) (as a function of $q$ for a fixed $n>0)$ on $q \in(p, r) \subset[0,1]$ we conclude that a solution $q^{\bullet} \in(p, r)$ of (15) exists for any $n>0$. Uniqueness of $q^{\bullet}$ would follow if the function $\Phi(q \mid n)$ is shown to be a non-increasing as a function of $q \in(p, r)$ as will be done below. (Note that the condition that $\Phi(q \mid n)$ is a non-increasing function - rather than being strictly decreasing - is sufficient in this case, since we are solving for a root of the equation $\Phi(q \mid n)=q$.$) Equivalently, it is sufficient to show that the reciprocal of \Phi(q \mid n)$ :

$$
\{\Phi(q \mid n)\}^{-1}=\frac{b(q \mid n)-a(q \mid n)}{m-a(q \mid n)}=1+\frac{b(q \mid n)-m}{m-a(q \mid n)}
$$

is a non-decreasing function of $q \in(p, r)$. The latter however follows immediately from the fact that $a(q \mid n)$ and $b(q \mid n)$ are strictly increasing functions of $q \in(p, r)$ (see (32) and (35), respectively). Hence, one can write $q^{\bullet}=q(n)$, where $q(n)$ is the unique solution of (15) for a fixed value of $n>0$. Continuity of the implicit function $q(n)$ now follows from the classical implicit function theorem (see, e.g., Flemming 1987) and the continuity of $\Phi(q \mid n)$ in both parameters $n$ and $q$ for $n>0$ and $q \in(p, r)$.

Note that from (37) (from (38)) we have that the function $\Phi(q \mid n)$ is right-continuous (leftcontinuous) as a function of $q$ for a fixed $n>0$ at $q=p$ (at $q=r)$, since $\Phi(q \mid n) \rightarrow 1$ $(\Phi(q \mid n) \rightarrow 0)$ as $q \downarrow p($ as $q \uparrow r)$ for any $n>0$. Hence, the unique solution $q(n)$ of the equation (15) may be obtained by using standard bisection methods with a (closed) starting interval $[p, r]$ for $q(n)$. Some basic properties of the function $q(n)$ are: (a) Its limiting value for $n \downarrow 0$ is provided by (18), (b) for $n \rightarrow \infty$ the value $q(n)$ follows from Eqs. $(20)-(21)$, and (c) its value for $n=1$ derived from $(22)$ and the location of the mode $m$ is:

$$
q(1)=\frac{r\left(m-a_{p}\right)+p\left(b_{r}-m\right)}{b_{r}-a_{p}} .
$$

For other values of $n$, the value of $q(n)$ needs to be solved numerically using $(15)$ and (16). 
Figure 9 provides an example of the function $q(n)$ for $p=0.10, r=1-p=0.90, a_{p}=6.5$, $m=7$ and $b_{r}=10.5$. These values of $p, a_{p}, m, r$ and $b_{r}$ are taken the same as those used in Figure 1 in Section 2. In Figure 9 the values of $q(0)$ (Eq. (18)), $q(1)($ Eq. $(39))$ and $q(\infty) \approx 0.244$ (calculated from Eqs. (20) and (21) using the bisection method) are presented. In addition, Figure 9 depicts the value of $q(2)$ (of $q(7)$ ) associated with the triangular distribution (the TSP distribution with parameter $n=7$ ) in Figure 1 calculated from Eqs. (15) and (16) applying a bisection method. Note, in particular, the rapid increase of $q(n)$ in Figure 9 for $n \in[0,2]$ and rather a mild one for $n \in[2, \infty)$. Figure 10 plots the lower bound function $a\{q(n) \mid n\}$ (Eq. (12)) and the upper bound function $b\{q(n) \mid n\}$ (Eq. (13)) associated with the values of $q(n)$ in Figure 9. The values $a_{p}$ and $b_{r}$ of the functions $a\{q(n) \mid n\}$ and $b\{q(n) \mid n\}$ as $n \downarrow 0$, and a nearly linear behavior of these functions as a function of $n$ for larger values are quite noteworthy in Figure 10. Notice that $a\{q(1) \mid 1\}=6$ and $b\{q(1) \mid 1\}=11$ are obtained directly from Eq. (22).

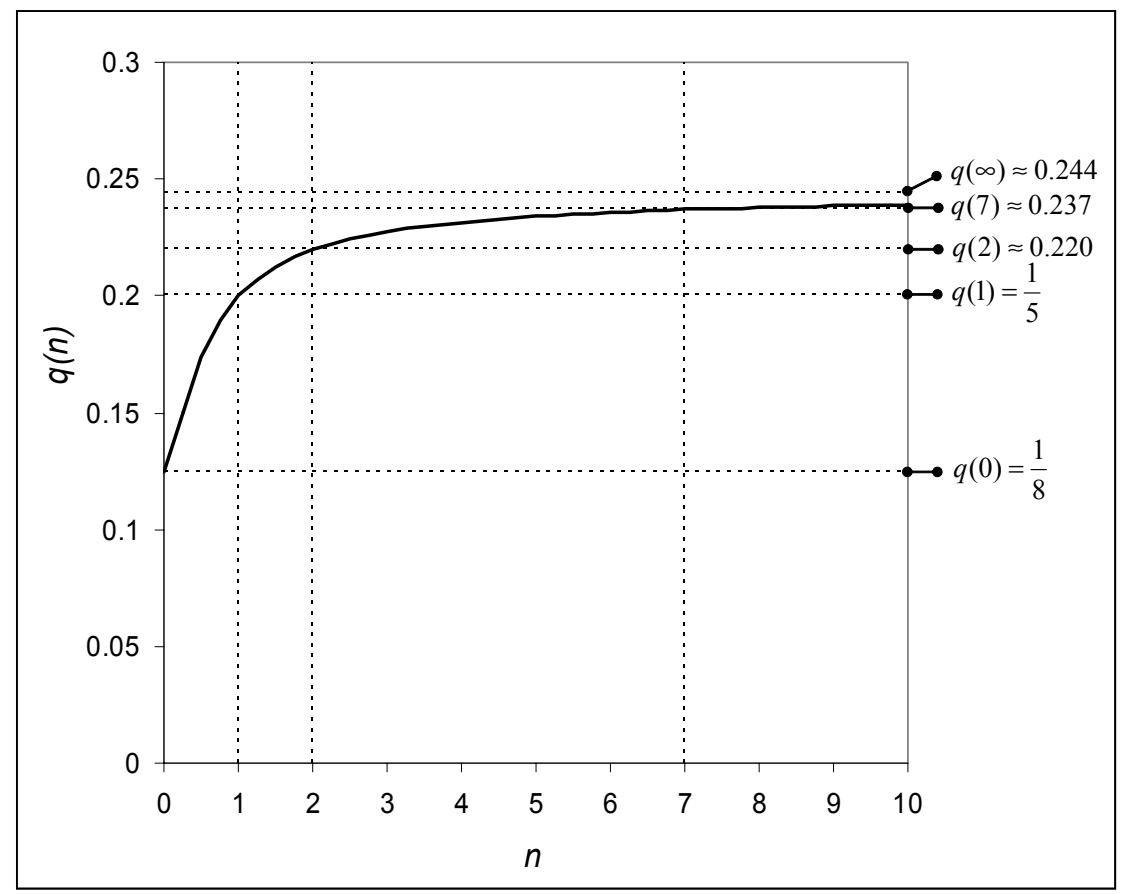

Figure 9. Graph of the implicit function $q(n)$ satisfying $(15)$ for the values

$$
p=0.10, r=1-p=0.90, a_{p}=6.5, m=7 \text { and } b_{r}=10.5 \text {. }
$$



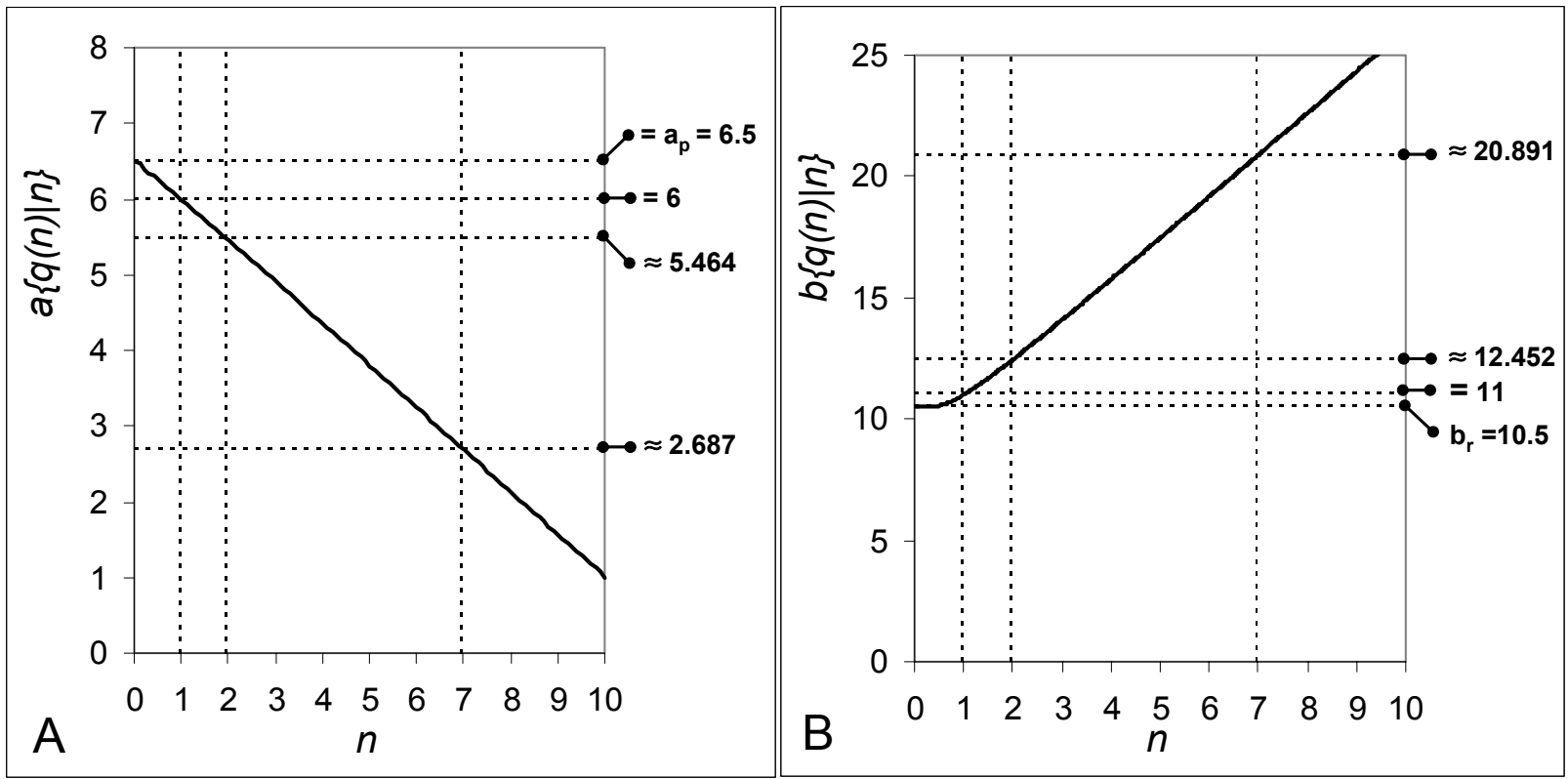

Figure 10. Graphs of the lower bound function $a\{q(n) \mid n\}$ (Eq. (12)) and the upper bound function $b\{q(n) \mid n\}$ (Eq. (13)), where $q(n)$ is the implicit function depicted in Figure 9.

\section{REFERENCES}

AbouRizk, S. M., Halpin D. W. and Wilson, J. R. (1992). Visual interactive fitting of beta distributions. Journal of Construction Engineering and Management, 117(4), 589-605.

Alexopoulos, C. and Seila, A.F. (1998). Output data analysis, in Handbook of Simulations (Chapter 7), Jerry Banks (Ed.), John Wiley \& Sons, New York, NY, pp. 225-272.

Altiok T. and Melamed, B. (2001). Simulation Modeling and Analysis with Arena, Cyber Research, Inc. and Enterprise Technologies, Inc.

Alpert, M. and Raiffa, H. (1982). A progress report on the training of probability assessors, in Judgment Under Uncertainty: Heuristics and Biases, D. Kahneman, Slovic, P. and Tversky, A. (Eds.), Cambridge University Press, New York, NY, pp. 294-305.

Banks, J., Carson, J.S., Nelson, B.L. and Nicol, D.M. (2005). Discrete-Event System Simulation (4th ed.), Prentice-Hall, Upper Saddle River, NJ.

Barabasi, A. (2003). Linked: How Everything Is Connected to Everything Else and What It Means (Reissue edition), Plume, New York, NY. 
Clark, C.E. (1962). The PERT model for the distribution of an activity. Operations Research, 10, 405406.

Cooke, R.M. (1991). Experts in Uncertainty, Oxford University Press, New York, NY.

Davidson, L.B. and Cooper, D.O. (1980). Implementing effective risk analysis at Getty Oil Company. Interfaces, 10, 62-75.

DeBrota, D. J., Roberts, S. D., Dittus, R. S. and Wilson J. R. (1989). Visual interactive fitting of bounded Johnson distributions. Simulation, 52, 199-205.

Elmaghraby, S.E. (1978). Activity Networks: Project Planning and Control by Network Models, John Wiley \& Sons, New York, NY.

Ferguson, T.S. (1973). A Bayesian analysis of some nonparametric problems. The Annals of Statistics, 1, 209-230.

Flemming, W. (1987). Functions of Several V ariables, Springer-Verlag, New York, NY.

Grubbs, F.E. (1962). Attempts to validate certain PERT statistics or a 'Picking on PERT'. Operations Research, 10, 912-915.

Herrerías, R., García, J. and Cruz, S. (2003). A note on the reasonableness of PERT hypotheses. Operations Research Letters, 31, pp. 60-62.

Johnson, D. (1997). The triangular distribution as a proxy for the beta distribution in risk analysis. The Statistician, 46 (3), 387-398.

Kamburowski, J. (1997). New validations of PERT times. Omega, International Journal of Management Science, 25 (3), 323-328.

Keefer, D.L. and Verdini A.V. (1993). Better estimation of PERT activity time parameters. Management Science, 39 (9), 1086 - 1091.

Kotz, S., Kozubowski, T.J. and Podgórski, K. (2001). The Laplace Distribution and Generalizations, Birkhäuser, Boston, MA.

Lau, H., Lau, A.H. and Ho C. (1998). Improved moment-estimation formulas using more than three subjective fractiles. Management Science, 44 (3), 346-350.

Law, A.M. and Kelton, W.D. (2000). Simulation Modeling and Analysis. McGraw-Hill, Boston, MA. 
Lindley, D. V. (1952). The theory of queues with a single server. Proceedings of the Cambridge Philosophical Society, 48, 277-289.

Malcolm, D.G., Roseboom, J.H. Clark, C.E. and Fazar W. (1959). Application of a technique for research and development program evaluation. Operations Research, 7, 646-649.

Moder, J.J. and Rodgers, E.G. (1968). Judgment estimate of the moments of PERT type distributions. Management Science, 18 (2), B76-B83.

Press, W.H., Flannery, B.P., Teukolsky, S.A. and Vettering, W.T. (1989). Numerical Recipes in Pascal: The Art of Scientific Computing. Cambridge University Press, Cambridge, UK.

Pulkkinen, U. and Simola, K. (2000). An Expert Panel Approach to Support Risk-Informed Decision Making, Sateiluturvakeskus (Radiation and Nuclear Safety Authority of Finland STUK), Technical report STUK-Y'TO-TR 129, Helsinki, Finland.

Selvidge, J.E. (1980). Assessing the extremes of probability distributions by the fractile method. Decision Sciences, 11, 493-502.

Simpson, T. (1755). A letter to the Right Honorable George Earl of Macclesfield, President of the Royal Society, on the advantage of taking the mean of a number of observations, in practical astronomy. Philosophical Transactions of the Royal Society of London, 49, 82-93.

Taggart, R. (1980). Ship Design and Construction, The Society of Naval Architects and Marine Engineers (SNAME), New York, NY.

Van Dorp, J.R., and Kotz S. (2002a). A novel extension of the triangular distribution and its parameter estimation. The Statistician, 51 (1), 63-79.

Van Dorp, J.R., and Kotz, S. (2002b). The standard two sided power distribution and its properties: with applications in financial engineering. The American Statistician, 56 (2), 90-99.

Vose D. (1996). Quantitative Risk Analysis, A Guide to Monte Carlo Simulation Modeling. John Wiley \& Sons, New York, NY.

Wagner, M. A. F. and Wilson, J. R. (1995). Graphical interactive simulation input modeling with bivariate Bézier distributions. ACM Transactions on Modeling and Computer Simulation, 5 (3), 163189. 
Wagner, M. A. F. and Wilson, J. R. (1996). Using univariate Bézier distributions to model simulation input processes. IIE Transactions, 28 (9), 699-711.

Welch, P.D. (1983). The statistical analysis of simulation results, in The Computer Performance Modeling Handbook, S. Lavenberg (Ed.), Academic Press, New York, NY, pp. 268-328.

Williams, T.M. (1992). Practical use of distributions in network analysis. Journal of Operations Research Society, 43 (3), 265-270.

Winston, W.L. (1993). Operations Research, Applications and Algorithms. Duxbury Press, Pacific Grove, CA. 


\section{BIOGRAPHICAL SKETCHES}

Samuel Kotz, PhD, is currently a visiting scholar at The George Washington University in Washington DC. Dr. Kotz received his $\mathrm{PhD}$ in mathematical statistics from Cornell University and has held distinguished visiting positions at Bucknell University, Bowling Green State University, Tel Aviv University, University of Guelph, Harbin Insititute of Technology (China), Luleå University (Sweden), and Copenhagen University. He was awarded honorary doctorates from the Harbin Institute of Technology in 1987, the University of Athens (Greece) in 1995, and from Bowling Green State University in 1996. In addition to serving as coordinating editor of Journal of Statistical Planning and Inference, Dr. Kotz has published over 120 papers and is the co-author with Professor N.L. Johnson (1917-2004) of the recently published Leading Personalities in Statistical Sciences and the classic four-volume Distributions in Statistics (now in its second edition), amongst his other books, monographs and compendia in statistics and probability.

Johan René van Dorp, DSc, is currently an Associate Professor at The George Washington University in Washington DC. Dr. van Dorp received his MSc. (1989) from the Delft University of Technology (The Netherlands) in Mathematics and Computer Science and his DSc. (1998) in Operations Research from The George Washington University. His research has resulted in 24 refereed journal publications dealing mainly (but not exclusively) with distribution theory, probabilistic risk assessment and reliability analysis. He serves as a reviewer for the European Journal of Operations Research, the Journal of Statistical Planning and Inference, Communications in Statistics, Metrika, Journal of Multivariate Analysis, Statistical Methods and Applications and Risk Analysis. 